Aus der Abteilung Kardiologie und Pneumologie

(Prof. Dr. med. G. Hasenfuß)

im Zentrum Innere Medizin

der Medizinischen Fakultät der Universität Göttingen

\title{
Die Herzfunktion während milder Hypothermie bei anästhesierten Schweinen: gesteigerte Inotropie auf Kosten einer diastolischen Dysfunktion
}

\author{
INAUGURAL - DISSERTATION \\ zur Erlangung des Doktorgrades \\ der Medizinischen Fakultät \\ der Georg-August-Universität zu Göttingen
}

\author{
vorgelegt von \\ Johannes Christoph \\ aus \\ Mühlhausen (Thür.)
}


Dekan:

I. Berichterstatter:

II. Berichterstatter:

III. Berichterstatter:

IV. Berichterstatterin:

Tag der mündlichen Prüfung:
Prof. Dr. med. C. Frömmel

Prof. Dr. med. Maier

Priv.-Doz. Dr. med. Tirilomis

Priv.-Doz. Dr. med. Kazmaier

Prof. Dr. rer. Nat. Virsik-Köpp

23.04.2012 


\section{Verzeichnis der Abkürzungen}
A.
Arteria / Arterie
$\alpha$
Proportionalitätsfaktor, welcher Konduktanz -
errechnetes Volumen mit dem HMV des Swan - Ganz -
Katheter in Beziehung setzt
$D_{\text {ed }} \quad$ enddiastolischer Druck
$D_{\text {es }}$
endsystolischen Druck
$\mathrm{dP} / \mathrm{dt}_{\max }$
maximale linksventrikuläre Druckanstiegsgeschwindigkeit
$\mathrm{dP} / \mathrm{dt}_{\min }$
maximale linksventrikuläre Druckabfallsgeschwindigkeit
Dtl.
Deutschland
EDDVB
linksventrikuläre enddiastolische Druck - Volumen -
Beziehung
ESDVB linksventrikuläre endsystolische Druck - Volumen -
Beziehung
$E_{\text {es }}$
Steigung der endsystolischen Druck - Volumen -
Beziehung
$\mathrm{Hb}$
Hämoglobingehalt
HF
Herzfrequenz
Hkt
Hämatokrit
HMV
Herzminutenvolumen
KHK
Koronare Herzkrankheit
$\mathrm{LVD}_{\text {ed }}$
enddiastolischer linksventrikulärer Druck
$L V D_{\text {es }}$
endsystolischer linksventrikulärer Druck
$\mathrm{MH}$
milde Hypothermie
msek
Millisekunden
$\mathrm{MVO}_{2}$
gemischt venöse Sauerstoffkonzentration
NL
Niederlande
PEEP
positiv endexspiratorischer Druck 


$\begin{array}{ll}\mathrm{pCO}_{2} & \text { arterieller Kohlenstoffdioxidpartialdruck } \\ \mathrm{pO}_{2} & \text { arterieller Sauerstoffpartialdruck } \\ \rho(\text { rho }) & \text { elektrische Leitfähigkeit des Blutes } \\ \mathrm{RT}_{50} & \text { Relaxation time - Relaxationszeit } \\ \mathrm{sO}_{2} & \text { arterielle Sauerstoffsättigung } \\ \mathrm{spm} & \text { Schläge pro Minute } \\ \mathrm{SV} & \text { linksventrikuläres Schlagvolumen } \\ \tau \text { (tau) } & \text { Zeitkonstante der isovolumetrischen linksventrikulären } \\ & \text { Relaxation } \\ \mathrm{t}_{\text {dia }} & \text { absolute Diastolendauer } \\ \mathrm{t}_{\text {sys }} & \text { absolute Systolendauer } \\ \mathrm{TTP} & \text { time to peak pressure - Zeit bis zur maximalen } \\ & \text { Kraftentwicklung } \\ \text { UK } & \text { Vereinigtes Königreich Großbritannien } \\ \text { US } & \text { Vereinigte Staaten von Amerika } \\ V_{\text {ed }} & \text { linksventrikuläres enddiastolisches Volumen } \\ V_{\text {es }} & \text { linksventrikuläres endsystolisches Volumen } \\ V_{\mathrm{P}} & \text { Parallelvolumen } \\ \text { V. cava inf. } & \text { Vena cava inferior } \\ \text { V. fem. } & \text { Vena femoralis } \\ \text { V. jug. int. } & \text { Vena jugularis interna } \\ \text { vs } & \text { versus } \\ \text { Vv. } & \text { Venae } \\ \text { Vv. fem. comm. } & \text { Venae femorales communes }\end{array}$




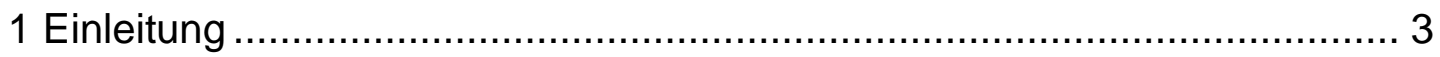

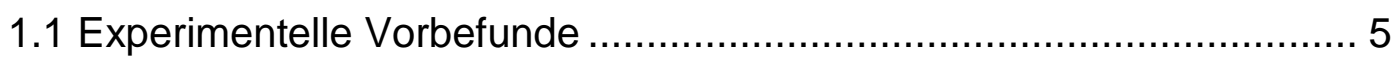

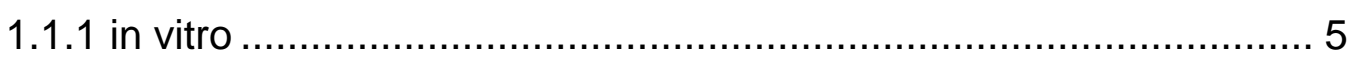

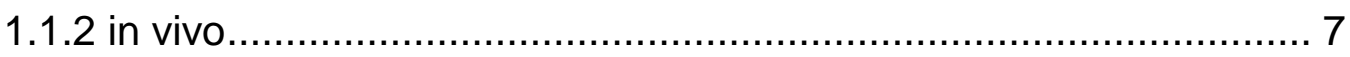

2 Material und Methoden ............................................................ 13

2.1 Tierexperimentelles Modell............................................... 13

2.1.1 Das Schwein als Versuchstier ........................................... 13

2.1.2 Versuchstiere .............................................................. 14

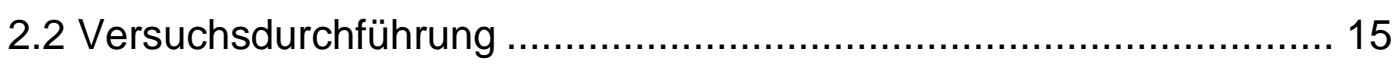

2.2.1 Narkoseeinleitung ........................................................ 15

2.2.2 Chirurgische Präparation .............................................. 17

2.2.3 Datenerfassung ......................................................... 19

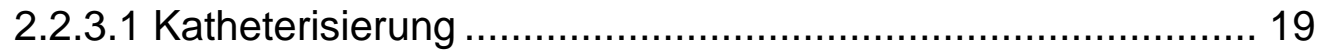

2.2.3.2 Konduktanz - Katheter - Prinzip ............................... 21

2.2.3.3 Narkoseführung .................................................. 23

2.2.3.4 Blutanalyse .............................................................. 23

2.2.3.5 Experimentelles Protokoll ......................................... 24

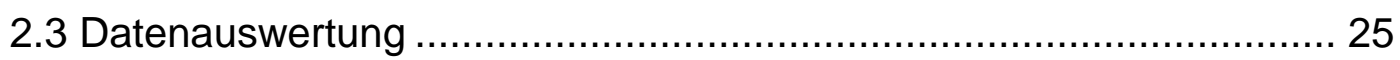

2.3.1 Linksventrikulärer Druck und Druckvolumenschleife ................. 26

2.3.2 Endsystolische und enddiastolische Druck - Volumen - Beziehung

$($ ESDVB und EDDVB) ....................................................... 28

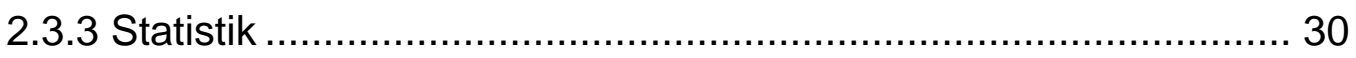

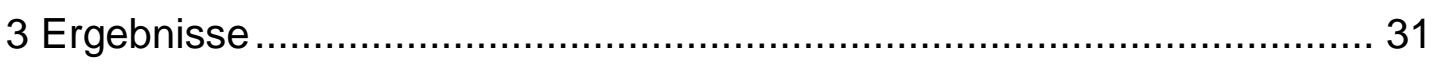

3.1 Linksventrikuläre Funktion und systemische Hämodynamik bei spontaner Herzfrequenz ........................................................ 33

3.2 Linksventrikuläre Funktion und systemische Hämodynamik unter Herzfrequenzsteigerung .............................................................. 37

3.3 Druck - Volumen - Beziehungen ..................................... 40

4 Diskussion............................................................................ 43 
4.1 Diskussion der Methode ........................................................... 43

4.2 Diskussion der Ergebnisse ................................................. 44

4.2.1 Metabolisches Gleichgewicht bei milder Hypothermie ............... 44

4.2.2 Kardiale Inotropie während $\mathrm{MH}$...................................... 45

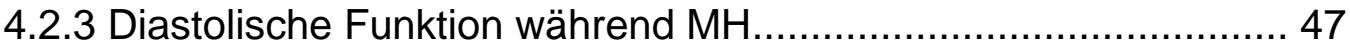

4.2.4 Potentielle subzelluläre Mechanismen der milden Hypothermie.. 49

4.3 Schlussfolgerung und klinische Anwendbarkeit............................ 51

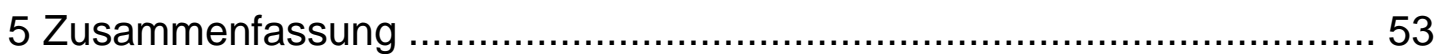

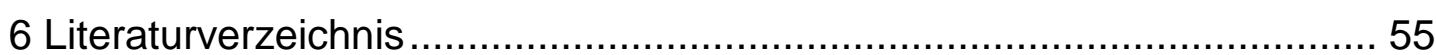

7 Publikation zum Inhalt der Dissertation ...............................................6 64 


\section{Einleitung}

Das Gehirn besitzt von allen Körpergeweben die geringste Toleranz gegenüber einer Ischämie. Daher zeigt bei Patienten nach plötzlichem HerzKreislaufstillstand und erfolgreicher Herz-Lungen-Wiederbelebung die Hirnfunktion die größte Einschränkung und das geringste Erholungsvermögen und limitiert somit den Erfolg einer Wiederbelebung. Zwei im Jahre 2002 veröffentlichte klinische Studien (Bernard et al. 2002; The hypothermia after cardiac arrest study group 2002) belegten eindrucksvoll, dass bei wiederbelebten Patienten nach Wiedereinsetzen des Kreislaufs die Induktion einer milden Hypothermie $\left(\mathrm{MH}, 32^{\circ} \mathrm{C}-34^{\circ} \mathrm{C}\right)$ für 12 bis $24 \mathrm{~h}$ die Ausprägung des hypoxischen Hirnschadens vermindern kann. Dies zeigte sich sowohl in einem geringeren neurologischen Defizit als auch in einem verbesserten Gesamtüberleben. Der Mechanismus der Neuroprotektion durch die MH nach Wiedereinsetzen der Perfusion, also nachdem die eigentliche Ischämie vorüber ist, ist zurzeit unklar. Diskutiert werden antiinflammatorische Mechanismen, die über eine verminderte Sauerstoffradikalfreisetzung maladaptive Reaktionen des reperfundierten Gewebes abschwächen (Zhao et al. 2007).

Die häufigste Ursache für einen plötzlichen Herzkreislaufstillstand ist kardial in Form eines akuten Myokardinfarktes oder eines plötzlichen Kammerflimmerns und ist fast immer Ausdruck einer vorbestehenden Herzerkrankung, in der Regel einer chronischen Herzinsuffizienz (Andresen 2005; Bardy et al. 2005). So steigt die Wahrscheinlichkeit, ein plötzliches Kammerflimmern zu erleiden, mit der Abnahme der Ejektionsfraktion als Parameter der systolischen Herzfunktion an (Klein 2006). Unterhalb einer Ejektionsfraktion von $35 \%$ wird bereits die präventive Implantation eines Defibrillators empfohlen (Jung et al. 2006). Das Szenario einer Reanimation 
setzt das Herz einer kompletten Ischämie von mehreren Minuten aus. Dieser Zeitraum ist anders als beim Gehirn nicht ausreichend, um zum ischämisch bedingten Untergang von Kardiomyozyten zu führen, bewirkt aber auch bei vollständig wiederhergestellter Myokarddurchblutung eine transiente kardiale Minderfunktion, das so genannte "stunning" (Heusch et al. 2005). Das reanimierte Herz ist also zusätzlich zu einer vorbestehenden Funktionseinschränkung akut geschwächt (Berg et al. 2008; Gonzalez et al. 2008; Kern et al. 1996).

In den oben genannten Studien wurde der Nutzen der milden Hypothermie bei Patienten mit unter intensivmedizinischer Therapie (Einsatz von Katecholaminen) und stabiler Kreislauffunktion belegt, während Patienten mit kritisch eingeschränkter Herzfunktion oder im kardiogenen Schock ausgeschlossen waren. Aufgrund der somit fehlenden klinischen Daten schließen die Leitlinien zur Reanimation den Einsatz der milden Hypothermie bei solchen Patienten aus und empfehlen den Einsatz der $\mathrm{MH}$ nur bei hämodynamisch stabilen Patienten (Nolan et al. 2005). Dieser Vorbehalt fußt zusätzlich auf dem Wissen, dass eine tiefere Hypothermie $\left(\leq 30^{\circ} \mathrm{C}\right)$ lebensbedrohliche Herzrhythmusstörungen auslösen kann und das Herzminutenvolumen absinken lässt (Nolan et al. 2003; Wolfrum et al. 2007). 


\subsection{Experimentelle Vorbefunde}

\subsection{1 in vitro}

Bereits 1897 beschrieb Langendorff die Temperatur als Determinante der Herzfunktion. Er stellte im isolierten Warmblüterherz fest, dass das Optimum der Kontraktionsstärke "[...] nicht etwa in die dem Warmblüterherzen adäquaten Temperaturen fällt, sondern tiefer als diese gelegen ist." (Langendorff 1897, S. 386).

Zahlreiche weitere Studien in verschiedenen Präparationen bestätigten diesen Effekt. Bei isolierten Papillarmuskelstreifen und Trabekeln von Ratten (Hiranandani et al. 2006; Liu et al. 1990; Shattock und Bers 1987), Kaninchen (Edman et al. 1974), Katzen (Templeton et al. 1974; Yeatman, Jr. et al. 1969), Schweinen (Weisser et al. 2001) und auch aus explantierten, nicht transplantierten humanen Spenderherzen (Weisser et al. 2001) steigerte eine Absenkung der Temperatur bereits um $2{ }^{\circ} \mathrm{C}$ die isometrisch entwickelte Kraft. Dies war charakteristischerweise mit einer Verlängerung der Einzelzuckung verbunden. Bei Papillarmuskelstreifen aus Ratten beispielsweise verlängert sich die Dauer einer Zuckung von $42^{\circ} \mathrm{C}$ nach $32^{\circ} \mathrm{C}$ um $100 \%$ bei einem Anstieg der isometrischen Kraft um ebenfalls $100 \%$. Diese Ergebnisse wurden auch im Modell des isolierten Herzens nachvollzogen. Bei isovolumetrischer Kontraktion von Hunde-, Ratten- und Kaninchenherzen erhöhte eine Hypothermie für eine gegebene Vorlast den systolisch entwickelten Druck (Monroe et al. 1964; Fukunami und Hearse 1989; Mattheussen et al. 1996). Ebenfalls stieg die Steilheit der endsystolischen Druck - Volumen - Beziehung an. Dieser Effekt zeigte sich in Untersuchungen an isolierten Herzen von Ratten (Fukunami und Hearse 1989), Guinea-Schweinen (Nakae et al. 2001), Kaninchen- (Mattheussen et al. 1996) und Hunden (Buckberg et al. 1977; Monroe et al. 1964; Suga et al. 
1988). In einer Arbeit der Gruppe um Suga et al. wurde bei isolierten Hundeherzen eine Drucksteigerung von $163 \mathrm{mmHg}$ auf $202 \mathrm{mmHg}$ bei gleich bleibendem endsystolischen Volumen und konstanter Nachlast demonstriert; dies entsprach einem Anstieg der Steigung der endsystolischen DruckVolumenbeziehung $\left(E_{e s}\right)$ um 24\% (Suga et al. 1988).

Anders als bei Stimulation durch Katecholamine, die eine Steigerung sowohl der entwickelten Kraft als auch der Kontraktionsgeschwindigkeit induzieren, geht die Erhöhung der isometrischen Kraftentwicklung während Hypothermie mit einer Verlängerung der Kontraktionsdauer und nicht oder nur kaum mit einer Beschleunigung der Kontraktion einher. Somit sind Kontraktilitätsparameter, die die Kontraktionsgeschwindigkeit reflektieren, wie z.B. das $\mathrm{dP} / \mathrm{dt}_{\max }$, während Hypothermie nicht notwendigerweise verändert. Parameter der Kontraktionskraft hingegen, wie z.B. die endsystolische Druck-Volumenbeziehung, sind prinzipiell eher geeignet, eine Steigerung der Inotropie während Hypothermie festzustellen. Diese Dichotomie zwischen Geschwindigkeit und Kraft der Kontraktion ist erstmals von Fukunami und Hearse benannt worden, die bei isolierten Rattenherzen während schrittweiser Abkühlung einen synchronen Anstieg von $E_{e s}$ und einen Abfall des $\mathrm{dP} / \mathrm{dt}_{\max }$ schilderten (Fukunami und Hearse 1989).

Analog zur verlängerten Kontraktionsdauer ist die Hypothermie auch durch eine verzögerte Relaxation gekennzeichnet. So nahm bei isolierten Herzen aus Schweinen (Stowe et al. 2007) und Kaninchen (Mattheussen et al. 1996) die maximale Druckabfallsgeschwindigkeit, $\mathrm{dP} / \mathrm{dt}_{\min }$, proportional zur Absenkung der Temperatur ab. Auch in der bereits zitierten Studie von Fukunami und Hearse an isolierten Rattenherzen fand sich über einen Temperaturbereich von $37^{\circ} \mathrm{C}$ bis $31^{\circ} \mathrm{C}$ eine Verminderung der maximalen Druckabfallsgeschwindigkeit um ca. $40 \%$.

Zusätzlich zur verlangsamten aktiven Relaxation in der frühen Diastole beschreiben einige Arbeiten auch eine Änderung der passiven 
Ventrikeleigenschaften im Sinne einer verminderten Compliance während Hypothermie. So zeigten Templeton et al. (1974) als auch Buckberg et al. (1977) bei isolierten Hundeherzen für gegebene enddiastolische Volumina mit Abnahme der Temperatur höhere Drücke. Andere Studien hingegen fanden diesen Effekt erst ab einer Temperatur $<30^{\circ} \mathrm{C}$ (Remensnyder und Austen 1965), oder berichteten keine Änderung (Monroe et al. 1964).

Zusammengefasst zeigen Studien in isolierten Präparationen, dass die $\mathrm{MH}$ systolisch die Kraftentwicklung bei gleich bleibender oder verminderter Kontraktionsgeschwindigkeit steigert, während die diastolische Funktion durch eine verlangsamte aktive Relaxation und wahrscheinlich auch durch eine verminderte Compliance beeinträchtigt wird.

\subsection{2 in vivo}

Angesichts der einheitlichen Befunde in vitro wäre zu erwarten, dass der positiv inotrope Effekt der Hypothermie auch in in-vivo-Modellen konsistent nachzuweisen ist. Tatsächlich aber ergibt die Literatur sowohl hinsichtlich der Messergebnisse als auch in Bezug auf die Interpretation der experimentellen Befunde ein uneinheitliches Bild.

Tabelle 1 gibt einen Überblick über Studien, die Änderungen des Herzminutenvolumens sowie der systolischen und diastolischen Funktion in vivo untersucht haben. 
Tabelle 1: HMV: Herzminutenvolumen; dP/dtmax: maximale linksventrikuläre Druckanstiegsgeschwindigkeit, dP/dtmin: maximale linksventrikuläre Druckabfallsgeschwindigkeit; tau: Zeitkonstante der isovolumetrischen Kontraktion; $E_{e s}$ : Steilheit der endsystolischen Druckvolumenbeziehung; PRSW: Steigung der Beziehung zwischen Vorlast und Schlagarbeit

\begin{tabular}{|c|c|c|c|c|c|c|c|}
\hline Autor & Jahr & Zitat & Spezies & Temperaturstufen & Anästhesie & $\begin{array}{l}\text { Kühlung/Thorax } \\
\text { offen - geschlossen }\end{array}$ & Ergebnis \\
\hline Goldberg & 1958 & $\begin{array}{l}\text { American Journal of } \\
\text { Physiology 194:92-99 }\end{array}$ & Hund & $\begin{array}{l}\text { Kühlung bis } \\
\text { Flimmern }\left(->19^{\circ} \mathrm{C}\right)\end{array}$ & Thiopental & $\begin{array}{ll}\text { Kühlung über } & \text { veno- } \\
\text { venösen } & \text { Shunt, } \\
\text { Thorakotomie } & \end{array}$ & $\begin{array}{l}\text { regionale } \\
\text { Arbeit } \uparrow, \\
\text { tau } \uparrow\end{array}$ \\
\hline $\begin{array}{l}\text { Remensnyder } \\
\text { und Austen }\end{array}$ & 1965 & $\begin{array}{l}\text { Journal of } \\
\text { and Cardiovascular } \\
\text { Surgery } \\
49: 339351\end{array}$ & Hund & $\begin{array}{l}\text { stufenweise } 37^{\circ}- \\
16^{\circ} \mathrm{C}\end{array}$ & Nembutal & $\begin{array}{l}\text { Kühlung über } \\
\text { kardiopulmonalen } \\
\text { Bypass, Thorakotomie }\end{array}$ & $\begin{array}{l}\text { Compliance } \\
\text { unter } 28^{\circ} \mathrm{C} \\
\text { vermindert }\end{array}$ \\
\hline $\begin{array}{l}\text { Rittenhouse } \\
\text { et al. }\end{array}$ & 1971 & $\begin{array}{l}\text { The Journal of } \\
\text { Thoracic and } \\
\text { Cardiovascular } \\
\text { Surgery 61/3:359-369 }\end{array}$ & Hund & $\begin{array}{l}38^{\circ} \mathrm{C}, 36^{\circ} \mathrm{C}, 34^{\circ} \mathrm{C}, \\
32^{\circ} \mathrm{C}, 30^{\circ} \mathrm{C}\end{array}$ & Surital & $\begin{array}{l}\text { Kühlung im Eisbad, } \\
\text { Hemithorakotomie }\end{array}$ & $\begin{array}{l}\mathrm{HMV} \downarrow \\
\mathrm{dP} / \mathrm{dtmax} \downarrow\end{array}$ \\
\hline $\begin{array}{l}\text { Boyer und } \\
\text { Gerstein }\end{array}$ & 1977 & $\begin{array}{l}\text { Journal of Thoracic } \\
\text { and Cardiovascular } \\
\text { Surgery } 74: 286-294\end{array}$ & Hund & $37^{\circ} \mathrm{C}+32^{\circ} \mathrm{C}$ & Pentobarbital & $\begin{array}{l}\text { Kühlung mittels } \\
\text { Thermodecken, } \\
\text { geschlossener Thorax }\end{array}$ & $\begin{array}{l}\mathrm{HMV} \downarrow \\
\mathrm{dP} / \mathrm{dtmax} \downarrow \\
\mathrm{dP} / \mathrm{dtmin} \downarrow\end{array}$ \\
\hline Greene et al. & 1989 & $\begin{array}{l}\text { Circulation } 80 \text { (Suppl } \\
\text { III) III } 44-48\end{array}$ & Schwein & $\begin{array}{l}38^{\circ} \mathrm{C}, 37^{\circ} \mathrm{C}, 36^{\circ} \mathrm{C}, \\
35^{\circ} \mathrm{C}, 34^{\circ} \mathrm{C}\end{array}$ & $\begin{array}{l}\text { a-chloralose, } \\
\text { Urethan }\end{array}$ & $\begin{array}{l}\text { Kühlung über } \\
\text { rechtsventikulären } \\
\text { Bypass, offener } \\
\text { Thorax }\end{array}$ & $\begin{array}{l}\text { tau } \uparrow, \\
\text { regionale } \\
\text { Arbeit } \downarrow\end{array}$ \\
\hline
\end{tabular}


Fortsetzung Tabelle 1

\begin{tabular}{|c|c|c|c|c|c|c|c|}
\hline Oung et al. & 1992 & $\begin{array}{l}\text { The Journal of } \\
\text { Trauma Vol 33, No.5: } \\
671-678\end{array}$ & Schwein & $\begin{array}{l}38.5^{\circ} \mathrm{C}, \quad 35^{\circ} \mathrm{C}, \\
30^{\circ} \mathrm{C}\end{array}$ & $\begin{array}{l}\text { Fentanyl, } \\
\text { Pancuroni- } \\
\text { um, Isofluran }\end{array}$ & $\begin{array}{l}\text { Kühlung über AV- } \\
\text { Shunt, geschlossener } \\
\text { Thorax }\end{array}$ & HMV $\downarrow$ \\
\hline Tveita et al. & 1994 & $\begin{array}{l}\text { Anest Analg 79: } \\
212.218\end{array}$ & Hund & $\begin{array}{l}37^{\circ} \mathrm{C}, 34^{\circ} \mathrm{C}, 31^{\circ} \mathrm{C}, \\
28^{\circ} \mathrm{C}\end{array}$ & Pentobarbital & $\begin{array}{l}\text { Wärmeaustauscher in } \\
\text { Ösophagus und } \\
\text { Enddarm, linksseitige } \\
\text { Thorakotomie }\end{array}$ & $\begin{array}{l}\mathrm{dP} / \mathrm{dtmax} \downarrow \\
\mathrm{HMV} \downarrow\end{array}$ \\
\hline Tveita et al. & 1998 & $\begin{array}{l}\text { Journal of Applied } \\
\text { Physiology } 85: 2135- \\
2139\end{array}$ & Hund & $37^{\circ} \mathrm{C}, 31^{\circ} \mathrm{C}, 25^{\circ} \mathrm{C}$ & Pentobarbital & $\begin{array}{l}\text { Wärmeaustauscher in } \\
\text { Ösophagus und } \\
\text { Enddarm, linksseitige } \\
\text { Thorakotomie }\end{array}$ & $\begin{array}{l}\text { dP/dtmax } \downarrow \text {, } \\
\text { dP/dtmin } \downarrow \text {, } \\
\text { tau^, } \\
\text { PRSW } \downarrow\end{array}$ \\
\hline Weisser et al. & 2001 & $\begin{array}{ll}\text { Basic Research } \\
\text { Cardiology }\end{array}$ & Schwein & $\begin{array}{l}37^{\circ} \mathrm{C}, 35^{\circ} \mathrm{C}, 33^{\circ} \mathrm{C} \\
31^{\circ} \mathrm{C}\end{array}$ & $\begin{array}{l}\text { Fentanyl, } \\
\text { Flunitraze- } \\
\text { pam, } \\
\text { Propofol, } \\
\text { Vecouronium }\end{array}$ & $\begin{array}{l}\text { Kühlung über } \\
\text { Kardiopulmonalen } \\
\text { Bypass, geschlossener } \\
\text { Thorax }\end{array}$ & $\begin{array}{l}\mathrm{HMV} \uparrow \\
\mathrm{dP} / \mathrm{dtmax} \uparrow\end{array}$ \\
\hline Lewis et al. & 2002 & $\begin{array}{l}\text { Journal of the } \\
\text { American College of } \\
\text { Cardiology 39: 102- } \\
108\end{array}$ & Mensch & $\begin{array}{l}37^{\circ} \mathrm{C}, 35^{\circ} \mathrm{C}, 33^{\circ} \mathrm{C} \\
31^{\circ} \mathrm{C}\end{array}$ & $\begin{array}{l}\text { Fentanyl, } \\
\text { Propofol }\end{array}$ & $\begin{array}{l}\text { Kühlung über } \\
\text { Kardiopulmonalen } \\
\text { Bypass, Swan-Ganz- } \\
\text { Katheter, Konduktanz- } \\
\text { Katheter, rechtsatrialer } \\
\text { Pacer }\end{array}$ & $\begin{array}{l}\text { Emax } \downarrow \text {, } \\
S W \downarrow \text {, time } \\
\text { to peak } \\
\text { ejektion } \uparrow\end{array}$ \\
\hline
\end{tabular}


Fortsetzung Tabelle 1

\begin{tabular}{|c|c|c|c|c|c|c|c|}
\hline $\begin{array}{l}\text { Perez-de-Sá } \\
\text { et al. }\end{array}$ & 2002 & $\begin{array}{l}\text { Anesthesiology } 97: \\
1189-1197\end{array}$ & Schwein & 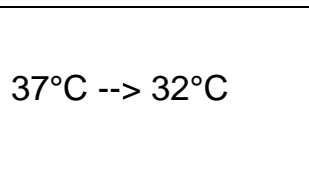 & $\begin{array}{l}\text { Fentanyl, } \\
\text { Vecouronium, } \\
\text { Midazolam }\end{array}$ & $\begin{array}{l}\text { Oberflächenkühlung } \\
\text { bei geschlossenem } \\
\text { Thorax }\end{array}$ & $\mathrm{HMV} \downarrow$ \\
\hline Dae et al. & 2003 & Stroke 34: 734-738 & Schwein & $38^{\circ} \mathrm{C}, 32^{\circ} \mathrm{C}$ & Isofluran & $\begin{array}{l}\text { Vena-cava- inferior - } \\
\text { Kühlkatheter, Open- } \\
\text { Chest für Ultraschall- } \\
\text { Flussmesskopf in } \\
\text { Aorta für HMV }\end{array}$ & $\mathrm{HMV}=$ \\
\hline $\begin{array}{l}\text { Boddicker et } \\
\text { al. }\end{array}$ & 2005 & $\begin{array}{l}\text { Circulation } \\
\text { 3195-3201 }\end{array}$ & Schwein & $\begin{array}{l}37^{\circ} \mathrm{C}, 35^{\circ} \mathrm{C}, 33^{\circ} \mathrm{C}, \\
30^{\circ} \mathrm{C}\end{array}$ & Halothan & $\begin{array}{l}\text { Swan-Ganz-Katheter } \\
\text { für HMV; Pacerkabel } \\
\text { zur Induktion VF, } \\
\text { Kühlung mittels } \\
\text { Eispackungen }\end{array}$ & $\mathrm{HMV} \downarrow$ \\
\hline $\begin{array}{l}\text { Nishimura et } \\
\text { al. }\end{array}$ & 2005 & $\begin{array}{l}\text { Interactive } \\
\text { CardioVascular and } \\
\text { Thoracic Surgery } 4: \\
101-105\end{array}$ & Hund & $37^{\circ} \mathrm{C}, 34^{\circ} \mathrm{C}, 32^{\circ} \mathrm{C}$ & $\begin{array}{l}\text { Phenobarbital, } \\
\text { Atropin, } \\
\text { Propanolol }\end{array}$ & $\begin{array}{l}\text { Thorakotomie, } \\
\text { Konduktanz-Katheter } \\
\text { und Mikromanometer } \\
\text { über Herz-Apex, } \\
\text { Kühlung im } \\
\text { Wasserbad mit Eis }\end{array}$ & $\begin{array}{l}E m a x \uparrow, \\
\operatorname{HMV}_{\downarrow}\end{array}$ \\
\hline Fischer et al. & 2005 & $\begin{array}{l}\text { Journal of } \\
\text { Investigative Surgery } \\
18: 291-295\end{array}$ & Hund & $37^{\circ} \mathrm{C}-->35^{\circ} \mathrm{C}$ & Thiopental & $\begin{array}{l}\text { spontane Auskühlung, } \\
\text { geschlossener Thorax }\end{array}$ & $\begin{array}{l}\mathrm{HMV} \downarrow \\
\operatorname{tau} \uparrow, \\
\mathrm{dP} / \mathrm{dtmin} \downarrow\end{array}$ \\
\hline
\end{tabular}


Bei allen aufgeführten Studien fiel während Hypothermie die Herzfrequenz ab und stieg, sofern berichtet, der systemische Widerstand an. Die Mehrzahl der Arbeiten zeigt einen Abfall des Herzminutenvolumens während Hypothermie, vor allem bei Temperaturen unterhalb von $31^{\circ} \mathrm{C}$. Das $\mathrm{dP} / \mathrm{dt}_{\max }$ fiel fast immer ab, während Kontraktilitätsparameter aus der Druckvolumenbeziehung stiegen, gleich blieben oder abfielen. Konsistent zeigen die Arbeiten hingegen eine verlangsamte Relaxation $\left(\mathrm{dP} / \mathrm{dt}_{\min }\right.$, Verlängerung des $\mathrm{T}$ ).

Keine der Arbeiten hat bisher zusammenhängend systolische und diastolische Funktion während Hypothermie untersucht, und auch enddiastolische Druckvolumenbeziehungen während $\mathrm{MH}$ sind in vivo bisher nicht beschrieben worden. Zudem sind häufig kardiodepressive Barbiturate zur Anästhesie verwendet worden, die bei verlangsamtem Metabolismus während Kühlung möglicherweise akkumulieren. Zudem ist eine Thorakotomie eine hochinvasive und über den Zeitverlauf tendenziell instabile Präparation. Schließlich sind die bisher überwiegend verwendeten Kühlmethoden (Eisbad, spontanes Abkühlen) unpräzise und erzeugen einen Temperaturgradienten von außen nach innen, welcher ein Kältezittern und dadurch eine erhöhte Stoffwechselaktivität hervorruft und weiterhin den Bedarf an Anästhetika erhöht. So schlussfolgerten die Mehrzahl der Arbeiten, dass die Hypothermie eher einen negativ inotropen Effekt ausübt und dieser zur Minderung des Herzzeitminutenvolumens beiträgt (Greene et al. 1989).

Ziel der vorliegenden Arbeit war es daher, ein möglichst kliniknahes Modell der Hypothermie zu nutzen, welches dem Vorgehen bei Patienten bestmöglich entspricht. Wir wählten ein Modell mit geschlossenem Thorax, einer Anästhesie, die so auch bei Patienten auf einer Intensivstation eingesetzt wird und benutzten einen intravasalen Kühlkatheter, mit dem die Körpertemperatur auf $0,1^{\circ} \mathrm{C}$ genau kontrolliert werden kann. Zur Messung diente ein Druckvolumenkatheter, der sämtliche oben genannten Parameter 
der Ventrikelfunktion erfasst. Die Messungen umfassten auch eine transiente Okklusion der V. cava inf., so dass lastunabhängige Parameter der Ventrikelfunktion bestimmt werden konnten. 


\section{Material und Methoden}

\subsection{Tierexperimentelles Modell}

\subsubsection{Das Schwein als Versuchstier}

Für die vorliegende Arbeit wurden Schweine als Versuchstiere gewählt. Aufgrund der Ähnlichkeit physiologischer und pathophysiologischer Vorgänge im Schwein und im Menschen können zu Grunde liegende Mechanismen fast direkt in die Humanmedizin übertragen werden. Dies gilt insbesondere für die Beurteilung kardiovaskulärer Parameter sowie das Verhalten des Organismus unter Reanimation. Zusätzlich sind die Reaktionen auf Arzneimittel, wie beispielsweise Isofluran oder Pancuronium, denen des Menschen sehr ähnlich. Somit bildet das tierexperimentelle Modell am Schwein eine wichtige Ressource sowohl in der biomedizinischen, als auch in der angewandten Forschung (Almond 1996).

Anders als im Hunde - oder Kleintiermodell hat man bei Schweinen mit einem Körpergewicht von 50-60 kg ein vergleichbares Verhältnis zwischen Herz und Körpergröße wie beim Menschen. Auch die Reaktion des Herzkreislaufsystems auf diverse Stimuli ist dem des Menschen ähnlich (Hannon et al. 1990). Somit können Ergebnisse einfacher auf die Klinik übertragen werden. Eine Untersuchung am isolierten Herzen, wie beispielsweise in der Arbeit von Fukunami und Hearse (1989), kann zwar die Messdeterminanten fast perfekt kontrollieren, ist jedoch nur bedingt klinisch relevant, da nur durch die integrative Betrachtung des gesamten HerzKreislaufsystems aussagekräftige Ergebnisse gewonnen werden können. 


\subsubsection{Versuchstiere}

Diese Studie wurde an zehn weiblichen, domestizierten Schweinen (Kreuzung deutscher Landrasse) mit einem Gewicht von $69 \pm 5 \mathrm{~kg}$ durchgeführt. Die Tiere wurden auf dem Landwirtschaftlichen Versuchsgut Relliehausen der Universität Göttingen gehalten und ein bis zwei Tage vor dem Versuch in das zentrale Tierlabor der Universitätsklinik Göttingen gebracht. Die Schweine wurden auf Stroh sowie in Gruppen von bis zu 3 Tieren gehalten und mit hofeigener Getreidemischung und Leitungswasser versorgt. Die Pflege der Tiere wurde von qualifiziertem Personal durchgeführt und tierärztlich überwacht.

Diese Studie wurde vom Niedersächsischen Landesamt für Verbraucherschutz und Lebensmittelsicherheit Braunschweig geprüft und genehmigt (Aktenzeichen der Tierversuchsgenehmigung: G307). Der Versuch entspricht dem "Guide for the Care and Use of Laboratory Animals“, welches vom US National Institute of Health heraus gegeben wurde (National Research Council 1996). 


\subsection{Versuchsdurchführung}

\subsubsection{Narkoseeinleitung}

Diese Versuchsreihe erfolgte in Kooperation mit der Abteilung HerzThoraxchirurgie (Prof. Schöndube, Dr. Schmitto, Rainer Holland) der Universität Göttingen.

Die sedierende Prämedikation erfolgte durch Injektion von $25 \mathrm{mg} / \mathrm{kg}$ Ketamin [Ketamin Inresa $10 \mathrm{ml} 500 \mathrm{mg} 50 \mathrm{mg} / \mathrm{ml}$, Inresa Arzneimittel GmbH, Dtl.] und $0.3 \mathrm{mg} / \mathrm{kg}$ Midazolam [Midazolam-ratiopharm $5 \mathrm{mg} / \mathrm{ml}$, ratiopharm $\mathrm{GmbH}$, Ulm, Dtl.] in die Nackenmuskulatur. Fakultativ wurden bei unzureichender Sedierung noch $10 \mathrm{mg} / \mathrm{kg}$ Ketamin nachinjiziert. Nach behutsamem Transport in den Operationssaal wurden die Tiere auf einem beheizten OP-Tisch in Rückenlage gebracht und die Vorder- sowie Hinterläufe mit Haltebändern am Tisch fixiert. Anschließend wurde die Vena auricularis caudalis des linken Ohres punktiert, um einen peripheren venösen Zugang zur Applikation des Injektionsnarkotikums zu schaffen. Die Narkose wurde mit $5 \mathrm{mg} / \mathrm{kg}$ Thiopental [Thiopental Inresa 0,5g, Inresa Arzneimittel $\mathrm{GmbH}$, Dtl.] eingeleitet.

Die Intubation erfolgte über eine Tracheotomie. Das hierzu erforderliche Freilegen der Trachea musste aufgrund der atemdepressiven Wirkung von Thiopental sehr rasch geschehen. Hierzu wurde mit dem elektrischen Messer [Erbotom ACC 430, Erbe Elektromedizin GmbH, Dtl.] ein medianer Hautschnitt längs des Halses angelegt und im Folgenden die Trachea stumpf frei präpariert. Durch einen Querschnitt zwischen zwei Knorpelspangen wurde ein Tubus [Portex® Trachealtubus, Smiths Medical, Watfort, UK] der Größe 7.5, 8.0 oder 8.5, je nach Größe der Trachea, in die Luftröhre eingeführt. Der Tubus wurde nach dem Blocken zusätzlichen mit zwei Ligaturen [Vicry $\Theta^{\circ}, 2 / 0,3 \times 45 \mathrm{~cm}$, Ethicon $\circledast$, Norderstedt, Dtl.] an der Trachea 
fixiert. Daraufhin wurden die Tiere mit einem volumenkontrollierten Beatmungsgerät [Ventilog 2, Dräger, Lübeck, Dtl.] mit einem Raumluft/Sauerstoffgemisch von 50/50\% bei einer Atemfrequenz von 20/min beatmet. Über das Beatmungsgerät wurden $0.5 \%$ Isofluran [Forane, Abbott $\mathrm{GmbH}$, Wiesbaden, Dtl.] supportiv appliziert. Der endexspiratorische Druck wurde zur Vermeidung von lagerungsbedingten Atelektasen bei $+5 \mathrm{mmHg}$ gehalten (PEEP). Das Zugvolumen wurde so angepasst, dass die exspiratorische Kohlendioxidkonzentration, welche durch ein Kapnometer [Capnodig, Dräger, Lübeck, Dtl.] ständig gemessen und angezeigt wurde, zwischen 4 und $4.5 \%$ lag. 


\subsubsection{Chirurgische Präparation}

Nach der Intubation wurden in den Logen parallel zur Trachea die Vv. jugulares internae dextra/sinistra sowie die Aa. carotides communes dextra/sinistra dargestellt. In alle 4 Gefäße wurden 8F-Schleusen [Avanti+, Cordis, Johnson\&Johnson, US] mit Hilfe der Seldinger - Technik eingebracht und mit Ligaturen fixiert. Die Schleusen dienten als venöser Zugang und für das spätere Einbringen verschiedener intravsakulärer Katheter.

Über den nun geschaffenen venösen Zugang wurde die Anästhesie intravenös fortgeführt. Die Tiere erhielten $0.5 \mathrm{mg} / \mathrm{kg} / \mathrm{h}$ Midazolam [Midazolam-ratiopharm 15mg/3ml, ratiopharm-GmbH, Ulm, Dtl.], 20 $\mu \mathrm{g} / \mathrm{kg} / \mathrm{h}$ Fentanyl [Fentanyl-Janssen, Janssen-Cilag $\mathrm{GmbH}$, Dtl.] und $0.1 \mathrm{mg} / \mathrm{kg} / \mathrm{h}$ Pancuronium [Pancuronium DeltaSelect, DeltaSelect $\mathrm{GmbH}$, Dreieich, Dtı.] jeweils über einen Perfusor [Perfusor Secura FT, B. Braun, Melsungen, DtI.]. Zusätzlich wurde ein Bolus von 10.000 I.E. Heparin [Heparin-Natrium 25000 I.E. $/ 5 \mathrm{ml}$, ratiopharm-GmbH, Ulm, Dtl.] verabreicht. Nach der Präparation wurde der Halssitus mit Kompressen austamponiert und die Haut über dem Situs mit Backhausklemmen adaptiert.

Den Tieren wurde während des Protokolls kontrolliert Volumen zugeführt. Über Infusomaten [Infusomat II, B. Braun, Melsungen, Dtl.] wurden 4\%ige Gelatinelösung [0.75 ml/kg/h Gelafundin 4\%, B. Braun, Melsungen, Dtl.] und isotone Elektrolytlösung $[2.25 \mathrm{ml} / \mathrm{kg} / \mathrm{h}$, Sterofundin, B. Braun, Melsungen, Dtl.] in einer Gesamtmenge von $3 \mathrm{ml} / \mathrm{kg} / \mathrm{h}$ infundiert. Die Elektrolytlösung enthielt Natrium, Kalium, Magnesium, Chlorid sowie Laktat und wurde zusätzlich mit 2.5\% Glucose [Glucose 40\%, B. Braun, Melsungen, Dtl.] angereichert.

Danach wurden die Vv. fem. comm. analog zu der Halspräparation freigelegt, großlumige 14F -Schleusen [14 F, St. Jude Medical, US] in die Gefäße eingeführt und diese wiederum mit Ligaturen fixiert. Die Wundhöhlen wurden austamponiert und die Haut mit Backhausklemmen adaptiert. An den 
Seitenarm jeder Femoralisschleuse wurde ein Heparinperfusor angeschlossen, der kontinuierlich 1.000 I.E./h Heparin infundierte, um Thrombembolien vorzubeugen. 


\subsubsection{Datenerfassung}

\subsubsection{Katheterisierung}

Durch die gelegten Schleusen wurden verschiedene Katheter in den Gefäßen, beziehungsweise im Herz unter Röntgenkontrolle [BV Pulsera, Philips, Amsterdam, NL] positioniert. Über eine V. jug. int. wurde ein Swan Ganz - Katheter [Pulmonalarterienkatheter CCO, Edwards Lifesciences, US] in die Pulmonalarterie eingebracht, welcher es nach dem Thermodilutionsprinzip über eine Heizwendel ermöglichte, das Herzzeitvolumen in Minutenintervallen zu erfassen [Vigilance I, Edwards Lifesciences, US]. Über die zweite V. jug. int. wurde eine Schrittmachersonde im rechten Vorhof positioniert, so dass eine kontrollierte Herzfrequenzsteigerung mittels externem Schrittmacher [Pacesetter 3077, Dr. Osypka GmbH, Rheinfelden-Herten, Dtl.] möglich war. Die Stimulation im rechten Vorhof gewährleistete einen physiologischen Erregungs- und Kontraktionsablauf des Herzens. Über die Schleuse in der rechten A. carotis communis wurde ein Druck - Volumen - Katheter [CA-71103-PL, 7F, 12 Elektroden, CardioDynamics, NL] in den linken Ventrikel vorgeschoben. Dieser Katheter erfasste den linksventrikulären Druck sowie zunächst unkalibriert das linksventrikuläre Volumen [Sigma-5 DF, CardioDynamics Leycom, Zoetermeer, NL]. Aus diesen Daten wurden Druck Volumenschleifen errechnet und graphisch dargestellt; die Position des Katheters wurde dann so lange modifiziert, bis die Druck - Volumen Schleifen stabil und artefaktfrei dargestellt wurden. 


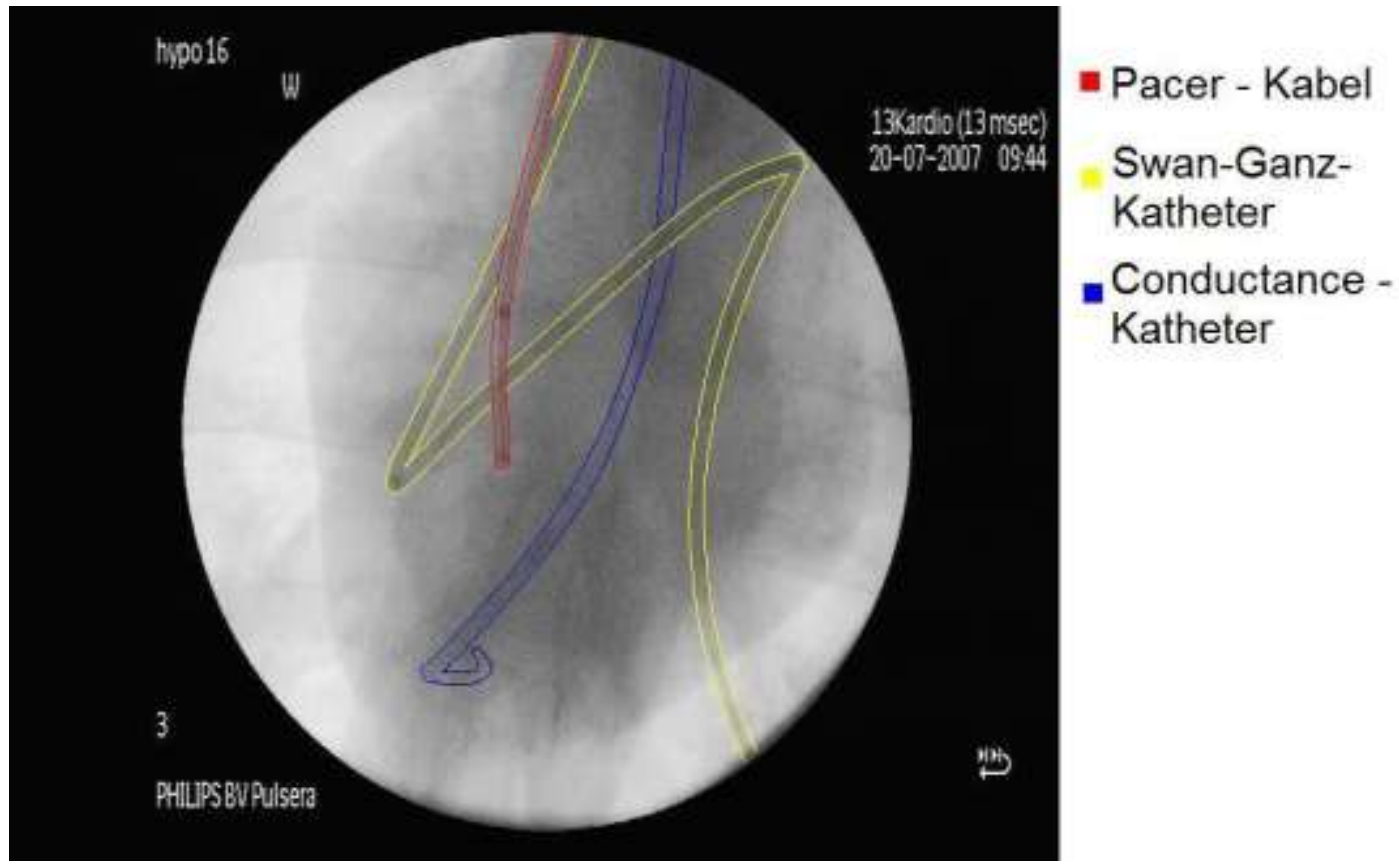

Abb.1: intraexperimentelle Röntgenaufnahme des instrumentierten Herzens

Über die linke V. femoralis wurde ein Ballonkatheter [NuMed, US] in die V. cava inferior eingeführt und auf Höhe des Zwerchfells positioniert. Der Ballon konnte kurzzeitig mit Kochsalzlösung entfaltet und die V. cava somit okkludiert werden; dies senkte akut den venösen Rückstrom zum Herzen ab und verminderte die Vorlast der Ventrikelkontraktion. Über die rechte V. fem. wurde ein intravasaler Kühlkatheter in die untere Hohlvene eingebracht. Dieser Katheter bestand aus einem dreifach gelappten, spiralgewickelten, Heparin - beschichteten Ballon, der aufgrund seiner Formgebung eine hohe Kontaktfläche mit dem Blutstrom in der V. cava. inf. hatte. Der Kühlkatheter wurde mit temperierter Kochsalzlösung aus einer Kühleinheit durchströmt [SetPoint ${ }^{\mathrm{TM}}$ System, Radiant Medical Inc., US]. Die Körpertemperatur wurde über eine Ösophagussonde ermittelt und die Leistung der Kühleinheit automatisch angepasst. Die Kühlflüssigkeit hatte dabei keinen direkten Kontakt mit dem Blut. In Abhängigkeit von der Größe des jeweiligen Versuchstieres lag die Kühlgeschwindigkeit bei ca. $1^{\circ} \mathrm{C}$ pro $30 \mathrm{~min}$. 


\subsubsection{Konduktanz - Katheter - Prinzip}

Um synchron Druck und Blutvolumen im linken Ventrikel erfassen zu können, wurde in der Versuchsreihe ein Konduktanzkatheter eingesetzt.

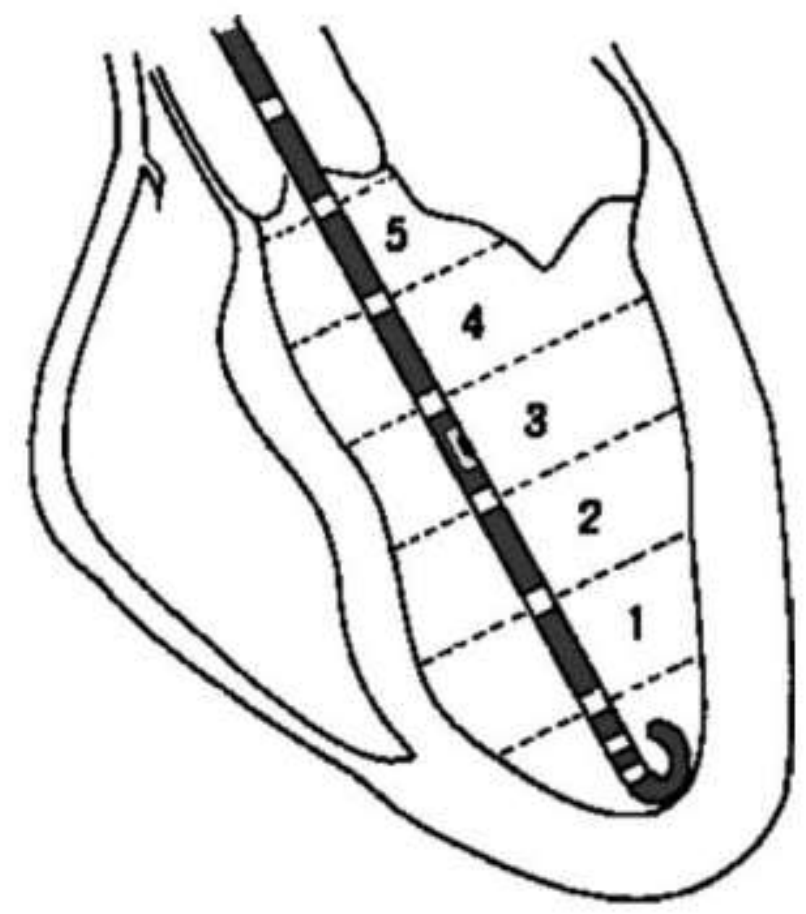

Abb. 2: Schemazeichnung eines Konduktanzkatheters. Über eine elektrische Membran (im Segement 3) wird der linksventrikuläre Druck erfasst. Die jeweils äußeren Elektroden erzeugen ein elektrisches Feld, welches über das intraventrikuläre Blut an die Segmentelektroden (1-5) weitergeleitet wird. Dabei ändert sich die Potentialstärke an den Elektroden proportional dem intraventrikulären Blutvolumen. (Abbildung aus Steendijk et al. 2004, S. D36)

Der Katheter wurde in der Längsachse des linken Ventrikels platziert. Um aus dem summierten Konduktanzsignal das absolute Volumen berechnen zu können, mussten Eichungen durchgeführt werden. Diese umfassten die Bestimmung der elektrischen Leitfähigkeit des Blutes $\rho$ (rho). Da sich dieser Wert über die Dauer des Versuches durch zugeführtes Volumen und mit der Temperatur ändern konnte, wurden den Tieren vor jeder Temperaturstufe 
$5 \mathrm{ml}$ arterielles Blut abgenommen, $\rho$ bestimmt und der Wert festgehalten. Strukturen, die dem linksventrikulären Blutvolumen anliegen (ventrikuläre Wand, rechter Ventrikel, Mediastinum), leiten ebenfalls das elektrische Feld und beeinflussen das Konduktanzsignal. Diese parallele Leitfähigkeit wurde als das so genannte Parallelvolumen $\left(V_{p}\right)$ mit der Methode der hypertonen Salinedilution (Baan et al. 1984) erfasst. Schließlich wird das tatsächliche Schlagvolumen durch die Konduktanzmethode tendenziell unterschätzt. Dies resultiert aus Inhomogenitäten im elektrischen Feld und der Tatsache, dass nicht die gesamte Längsachse des Ventrikels von den Segmenten abgedeckt wird. Diese Abweichung vom tatsächlichen Volumen wird durch den Faktor $\alpha$ ausgedrückt, welcher das durch den Konduktanzkatheter bestimmte HMV mit dem durch den Swan-Ganz-Katheter bestimmten Referenz - HMV in Beziehung setzt (Steendijk und Baan 2000).

Über den Konduktanzkatheter wurde weiterhin ein intraventrikuläres EKG aufgezeichnet. Die Datenerfassung erfolgte mit einer Auflösung von $250 \mathrm{~Hz}$. Die kalibrierte Auswertung des Ventrikelvolumens erfolgte offline nach Beendigung des Experimentes. 


\subsubsection{Narkoseführung}

Während des Versuches wurde das Atemzugvolumen bei konstanter Atemfrequenz so variiert, dass die exspiratorische $\mathrm{CO}_{2}$-Konzentration 4 bis 4,5 Vol\% betrug. Die Infusionsraten von Fentanyl und Midazolam wurden so gewählt, dass die spontane Herzfrequenz $<100$ /min lag und die Tiere auf Schmerzreiz (Klemmen einer Hautfalte) nicht reagierten. Erst dann wurde die muskuläre Relaxation begonnen.

\subsubsection{Blutanalyse}

Die gewonnenen Blutproben wurden direkt nach Entnahme in einem Blutgasanalysegerät (RapidLab 865, Bayer, Deutschland) analysiert. Dabei wurden die Parameter Sauerstoffsättigung $\left(\mathrm{sO}_{2}\right)$, Partialdruck für Sauerstoff $\left(\mathrm{pO}_{2}\right)$ und Kohlenstoffdioxid $\left(\mathrm{pCO}_{2}\right), \mathrm{pH}-$ Wert $(\mathrm{pH})$, Hämoglobingehalt $(\mathrm{Hb})$, Hämatokrit (Hkt), Sauerstoffgehalt, Natrium, Kalium, Kalzium sowie Glukose- und Laktatkonzentrationen erfasst. 


\subsubsection{Experimentelles Protokoll}

Nach Abschluss der Präparation erfolgte eine Pause von 30 min, in denen sich die hämodynamischen Parameter stabilisieren konnten.

Ziel des experimentellen Protokolls war die Analyse der Herzfunktion bei Normothermie $\left(37^{\circ} \mathrm{C}\right)$ und graduierter Hypothermie $\left(35^{\circ} \mathrm{C}, 33^{\circ} \mathrm{C}\right.$ und $\left.32^{\circ} \mathrm{C}\right)$. Dieser Temperaturbereich entsprach dem Regelungsbereich des Kühlaggregats. Bei jeder Temperaturstufe erfolgten zunächst Eichungen mit Bestimmung von $\rho, V_{p}$ und $\alpha$. Es folgten Messungen bei spontaner Herzfrequenz. Diese umfassten die steady-state Hämodynamik über 2 komplette Atemzyklen und Abnahme je einer systemisch-arteriellen und pulmonal-arteriellen Blutgasanalyse. Daran schloss sich die Aufzeichnung mehrerer kurzer V.-cava-inf.-Okklusionen an. Hierbei wurde das Atemzugvolumen kurzzeitig reduziert, um atemabhängige Vorlaständerungen zu vermeiden. Zusätzlich wurde bei $37^{\circ} \mathrm{C}$ und $33^{\circ} \mathrm{C}$ die Herzfrequenz mittels rechtsatrialer Schrittmacherstimulation auf 100/min, 125/min, 150/min und 175/min erhöht, solange jedem elektrischen Stimulus eine linksventrikuläre Kontraktion folgte. Die maximal erzielbare Herzfrequenz nahm bei Hypothermie charakteristischerweise ab.

Nach Abschluss des Protokolls wurden die Tiere in Narkose mit einer Bolusinfusion von $80 \mathrm{mMol}$ Kaliumchlorid getötet. 


\subsection{Datenauswertung}

Die Daten des Konduktanzkatheters wurden offline im Anschluss an die Experimente durchgeführt und mit Hilfe der CircLab@ - Software (freundlicherweise überlassen von Prof. Paul Steendijk, Abt. für Kardiologie, Universität Leiden, NL) ausgewertet.

Die berechneten hämodynamischen Parameter umfassten die Herzfrequenz $(H F)$, die absolute und relative Dauer von Systole ( $\left.t_{\text {sys }}(m s e k), t_{\text {sys }} \%\right)$ und Diastole ( $t_{\text {dia }}$ (msek), $\left.t_{\text {dia }} \%\right)$, den enddiastolischen $\left(L V D_{\text {ed }}\right)$ und endsystolischen $\left(L V D_{\text {es }}\right)$ linksventrikulären Druck, die maximale linksventrikuläre Druckanstiegs- und Abfallsgeschwindigkeit ( $\mathrm{dP} / \mathrm{dt}_{\max }$; $\mathrm{dP} / \mathrm{dt}_{\min }$ ), die Zeitkonstante der isovolumetrischen linksventrikulären Relaxation (tau, T (msek)) und das enddiastolische sowie endsystolische Volumen $\left(\mathrm{V}_{\mathrm{ed}}\right.$ und $\left.\mathrm{V}_{\mathrm{es}}\right)$. Der Zeitpunkt der End-Diastole wurde als der rechte untere, der Zeitpunkt der End-Systole als der linke obere Wendepunkt der Druckvolumenschleife bestimmt (s.u.).

Das Schlagvolumen (SV) wurde als $\mathrm{V}_{\mathrm{ed}}-\mathrm{V}_{\mathrm{es}}$ und das Herzminutenvolumen (HMV) als HF.SV berechnet. Der Gesamtkörper- Sauerstoffverbrauch $\left(\mathrm{MVO}_{2}\right)$ ergab sich aus der Differenz zwischen aortalem und pulmonalarteriellem Sauerstoffgehalt multipliziert mit dem HMV.

Alle erfassten Blutmesswerte wurden im Blutgasanalysegerät auf die jeweilige Temperatur korrigiert. 


\subsubsection{Linksventrikulärer Druck und Druckvolumenschleife}

Abbildung 3 veranschaulicht die Bestimmung von $\mathrm{dP} / \mathrm{dt}_{\max }, \mathrm{dP} / \mathrm{dt}_{\min }$ und $\mathrm{T}$ aus dem linksventrikulären Drucksignal. Diese Parameter repräsentieren die invivo-Korrelate der in vitro gemessenen Kontraktions- und Relaxationsgeschwindigkeiten und sind Kennzeichen der Inotropie ( $\mathrm{dP} / \mathrm{dt}_{\max }$ ) und der aktiven Relaxation in der frühen Diastole $\left(\mathrm{dP} / \mathrm{dt}_{\min }\right.$ und tau). Hervorzuheben ist, dass die genannten Parameter zeitabhängig sind und die Dimension linksventrikuläres Volumen nicht berücksichtigen. Ebenso spiegeln diese Parameter passive Eigenschaften des linken Ventrikels (Compliance) nicht wider.

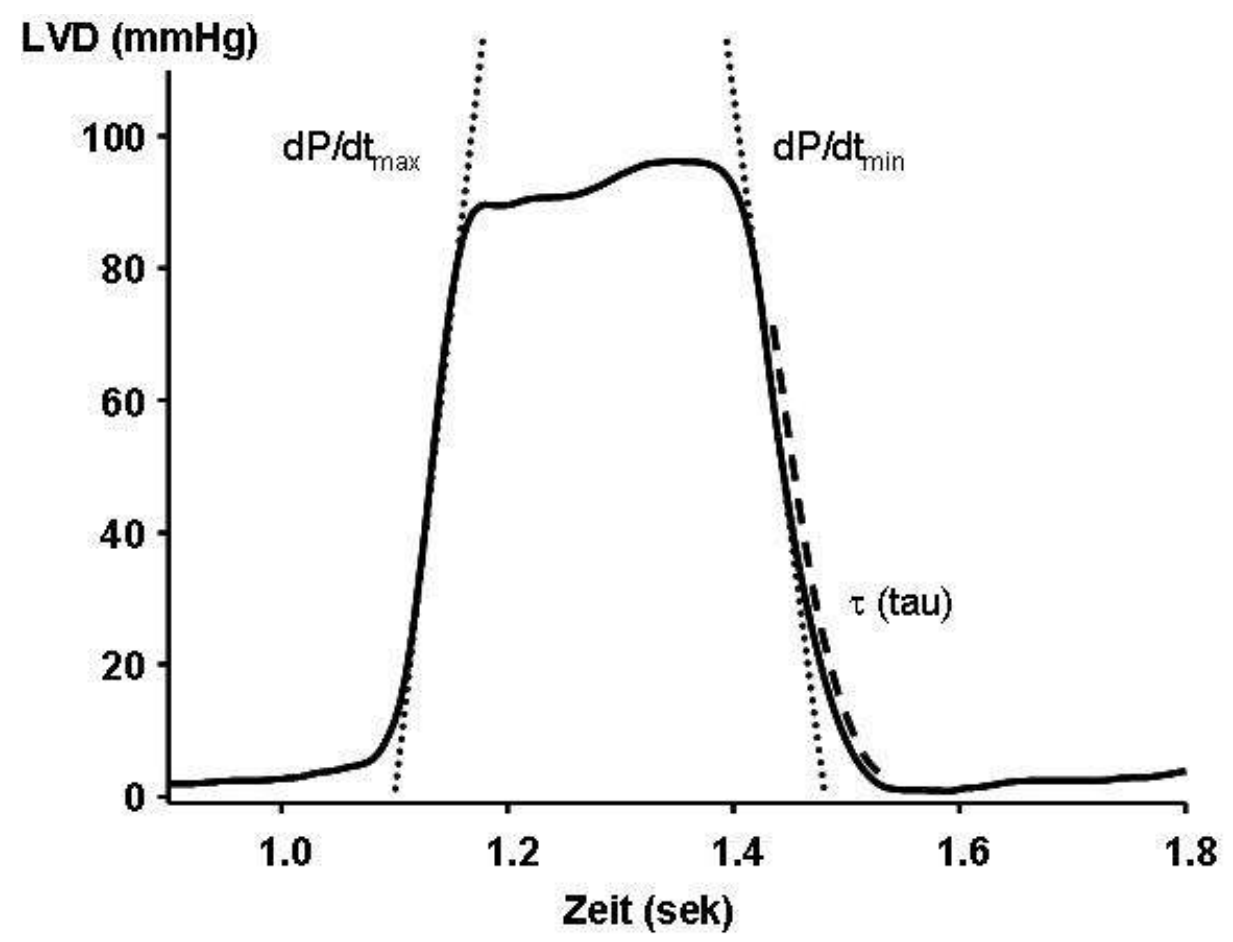

Abb.3: Bestimmung von $d P / d t_{\max }, d P / d t_{\min }$ und tau aus dem LVD. 
Abbildung 4 zeigt eine typische linksventrikuläre Druckvolumenschleife. Der endsystolische Quotient aus Druck und Volumen stellt ein in-vivo-Korrelat für die in vitro gemessene maximale Kontraktionskraft dar. Der enddiastolische Druck-Volumenquotient reflektiert denjenigen Zeitpunkt im Herzzyklus, an dem bei genügend schneller aktiver Relaxation sämtliche Prozesse der elektromechanischen Kopplung abgeschlossen sind und spiegelt somit am ehesten passive Eigenschaften des linken Ventrikels in vivo wider. Die Dimension Zeit und damit die Kontraktions- und Relaxationsgeschwindigkeiten bleiben in dieser Darstellung unberücksichtigt.

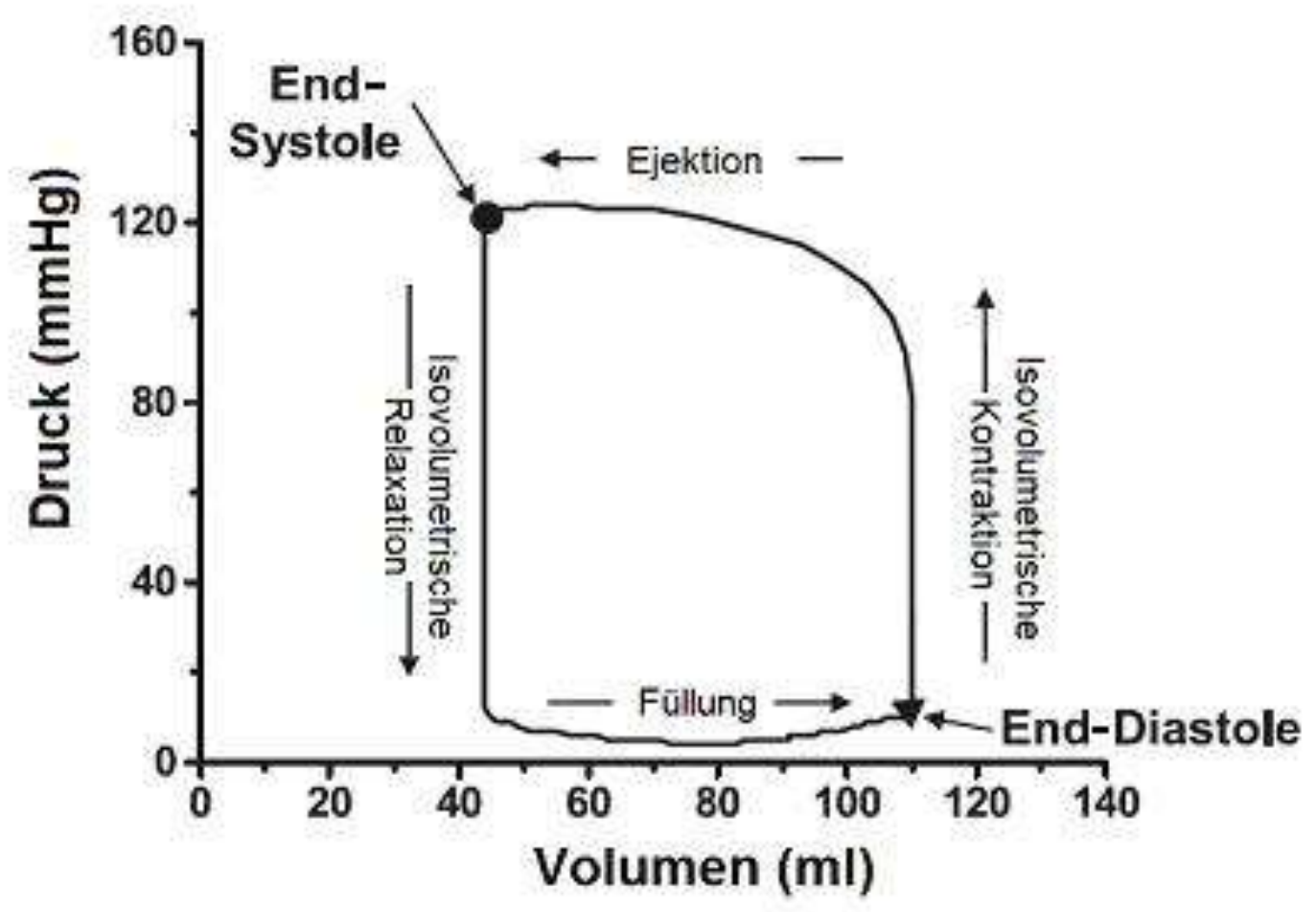

Abb. 4: Schema einer linksventrikulären Druckvolumenschleife (Abbildung modifiziert aus Burkhoff et al. 2005, S. H502) 


\subsubsection{Endsystolische und enddiastolische Druck - Volumen - Beziehung (ESDVB und EDDVB)}

Werden Druckvolumenschleifen während akuter Laständerung, d.h. vor Einsetzen des Baroreflexes aufgezeichnet, so lassen sich endsystolische und enddiastolische Druck-Volumen-Beziehungen herleiten (Burkhoff et al. 2005). Dies veranschaulicht Abbildung 5:

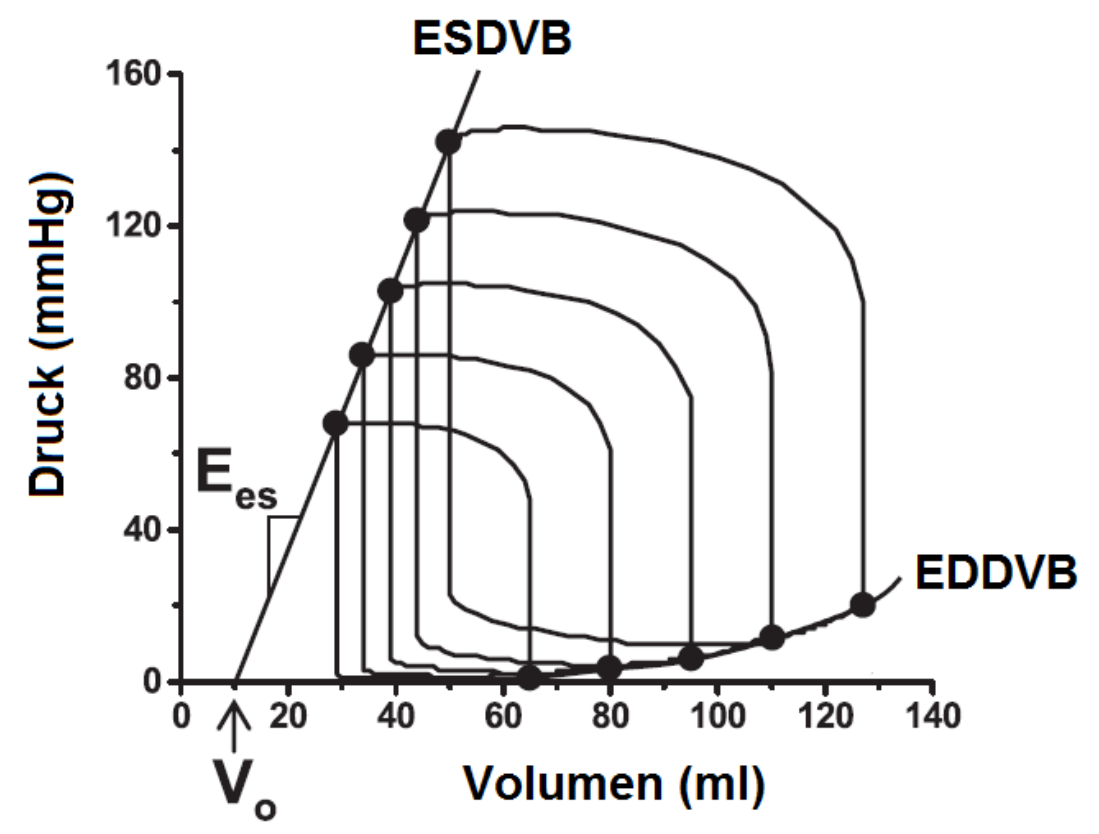

Abb.5: Schemazeichnung eines Druck-Volumen-Diagramms bei Vorlastwechselmanöver; ESDVB: endsystolische Druck-Volumen-Beziehung; EDDVB: enddiastolische Druck-Volumen-Beziehung; $E_{e s}$ : Steigung der ESDVB (Abbildung modifiziert aus Burkhoff et al. 2005, S. H502)

Die Position und Steilheit der jeweiligen Beziehungen sind Parameter der Inotropie (ESDVB), bzw. der Compliance (EDDVB) (Burkhoff et al. 2005). In der hier vorgestellten Studie wurde eine akute Laständerung durch Inflation des Ballonkatheters in der V. cava inf. herbeigeführt. Wir berechneten pro Messzeitpunkt anhand der linearen Geradengleichung $(y=a x+b)$ Achsenabschnitt und Steigung der jeweiligen ESDVB und EDDVB. Wie 
Burkhoff et al. (2005) ausführten, sind diese Beziehungen in vivo nur im physiologischen Druckbereich relevant, während sie bei pathophysiologisch niedrigen und hohen Ventrikeldrücken curvilinear verlaufen. Um die Position der Beziehungen im physiologisch bedeutsamen Bereich vergleichen zu können, berechneten wir zusätzlich aus den Gleichungen das endsystolische Volumen für einen endsystolischen Druck von $120 \mathrm{mmHg}\left(\mathrm{V}_{\mathrm{es}} 120\right)$ und das enddiastolische Volumen für einen enddiastolischen Druck von $10 \mathrm{mmHg}$ $\left(V_{\text {ed }} 10\right)$. 


\subsubsection{Statistik}

Alle Daten sind als Mittelwert \pm Standardfehler angegeben. Wir analysierten zuerst die Hämodynamik bei spontaner Herzfrequenz bei den Temperaturstufen $37^{\circ} \mathrm{C}, 35^{\circ} \mathrm{C}, 33^{\circ} \mathrm{C}$ und $32^{\circ} \mathrm{C}$. Hierzu wurde eine 1 -Wege Varianzanalyse für wiederholte Messungen angewandt. Einzelne Mittelwerte wurden mit Tukey's post-hoc-Test verglichen. Änderungen der Parameter während rechtsatrialer Schrittmacherstimulation wurden zwischen $37^{\circ} \mathrm{C}$ und $33^{\circ} \mathrm{C}$ mit einer 2-Wege-Varianzanalyse für wiederholte Messungen analysiert, wiederum mit Vergleich einzelner Mittelwerte mittels Tukey's posthoc-Test. Signifikanz wurde bei einem $p<0.05$ angenommen. Die Datenerhebung erfolgte mit der Tabellenkalkulationssoftware MS Excel, die statistische Auswertung mittels SigmaStat $\circledast$ Version 2.01., die Anfertigung der Graphen mit SigmaPlot@ Version 9.01 und MS Powerpoint. 


\section{Ergebnisse}

Während des Protokolls kam es zu keinen spontanen Arrhythmien oder Kreislaufinstabilitäten. Die Parallelvolumina $\mathrm{V}_{\mathrm{p}}(83 \pm 7 \mathrm{ml}, 86 \pm 7 \mathrm{ml}, 82 \pm 7 \mathrm{ml}$, $86 \pm 8 \mathrm{ml})$, der Korrekturfaktor $\alpha(0.45 \pm 0.03,0.43 \pm 0.03,0.43 \pm 0.03$ und $0.42 \pm 0.03)$ und die Blutleitfähigkeit $\rho(132 \pm 4,128 \pm 4,126 \pm 4$ und $129 \pm 6 \mathrm{~S} / \mathrm{m})$ unterschieden sich zwischen den Temperaturstufen $37^{\circ} \mathrm{C}, 35^{\circ} \mathrm{C}, 33^{\circ} \mathrm{C}$ und $32^{\circ} \mathrm{C}$ nicht.
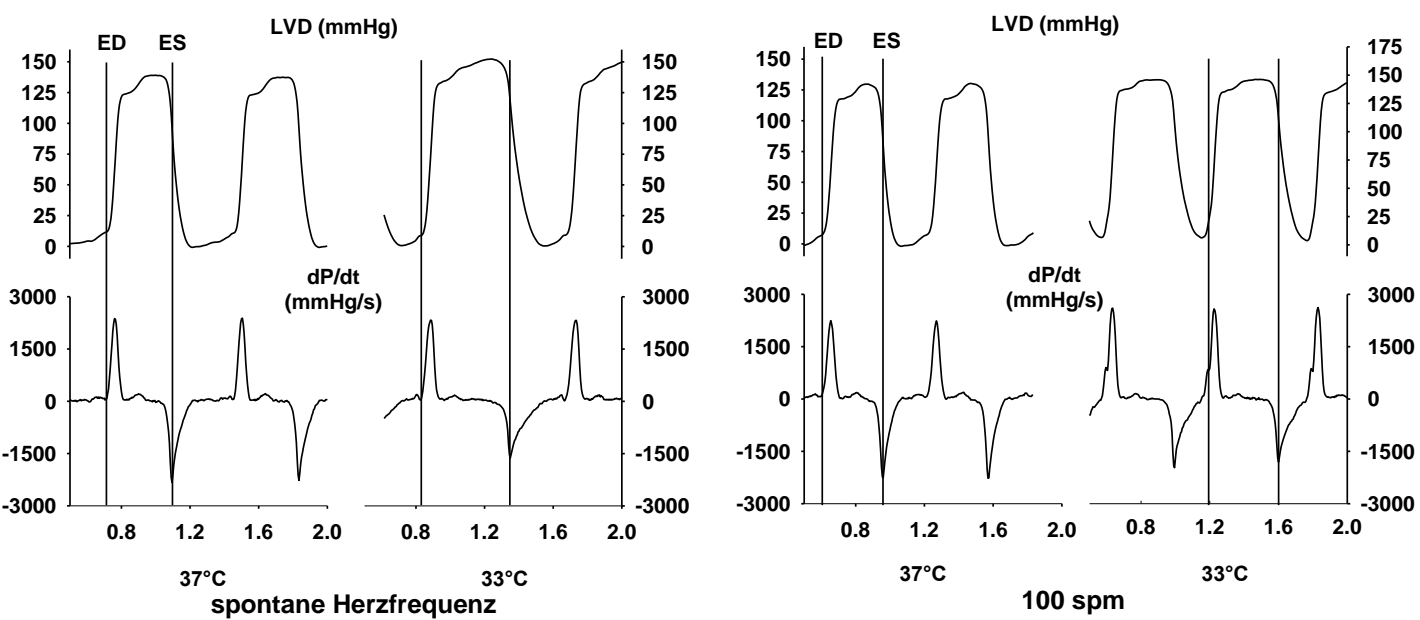

Abb. 6: Originalregistrierung des $L V D$ und des $d P / d t$ bei $37^{\circ} \mathrm{C}$ und $33^{\circ} \mathrm{C}$ bei spontaner Herzfrequenz (links) und bei rechtsatrialem Pacing mit 100/min (rechts). ED: End-Diastole, ES: End-Systole

Abbildung 6 zeigt typische Änderungen des linksventrikulären Drucks und $\mathrm{dP} / \mathrm{dt}$ während Hypothermie. Die spontane Herzfrequenz nimmt ab, wobei sich, anders als bei einer Ruhebradykardie, die Systolendauer verlängert. Das $\mathrm{dP} / \mathrm{dt}_{\max }$ bleibt unverändert, während sich das $\mathrm{dP} / \mathrm{dt}_{\min }$ verringert und das T verlängert, erkennbar am verlangsamten LVD-Abfall nach der EndSystole. Durch Schrittmacherstimulation wird die Diastolendauer weiter 
verkürzt, so dass die Relaxation des linken Ventrikels unvollständig bleibt und der enddiastolische Druck zunimmt. Diese Änderungen sind nachfolgend quantitativ ausgewertet. 


\subsection{Linksventrikuläre Funktion und systemische Hämodynamik bei spontaner Herzfrequenz}
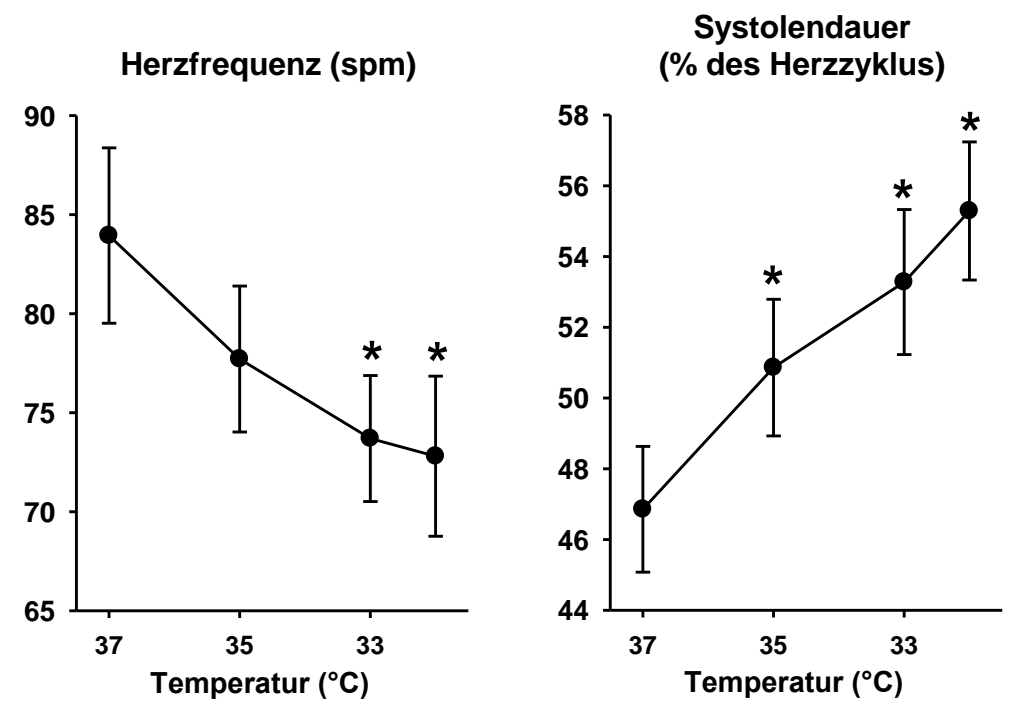

Abb. 7: mit sinkender Temperatur signifikanter Abfall der Herzfrequenz und Verlängerung der relativen Systolendauer

Die spontane Herzfrequenz verringerte sich mit Abfall der Körpertemperatur. Gleichzeitig nahm der Anteil der Systole am Herzzyklus zu (Abb.7). Quantitativ blieb die Dauer der Diastole trotz der Bradykardie nahezu konstant (Tabelle 2). 

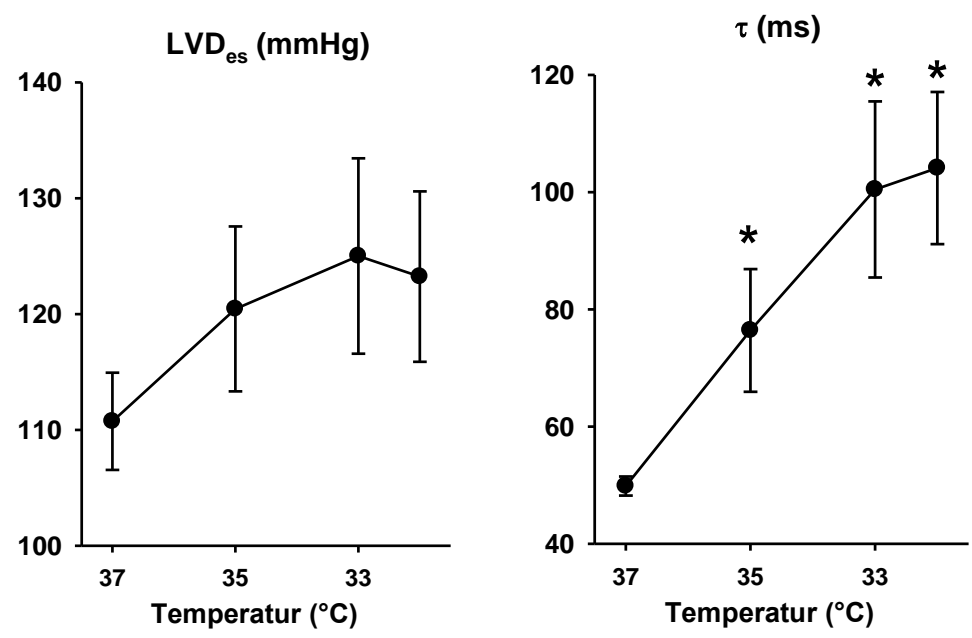

Abb. 8: $L V D_{e s}$ : endsystolischer linksventrikulärer Druck

r: Zeitkonstante der isovolumetrischen linksventr. Relaxation

Der endsystolische Druck änderte sich während Hypothermie nicht signifikant, während der enddiastolische Druck bei $32^{\circ} \mathrm{C}$ leicht zunahm (Tabelle 2). Die Zeitkonstante der isovolumetrischen Relaxation t nahm bereits beim Sprung von $37^{\circ} \mathrm{C}$ auf $35^{\circ} \mathrm{C}$ deutlich zu.
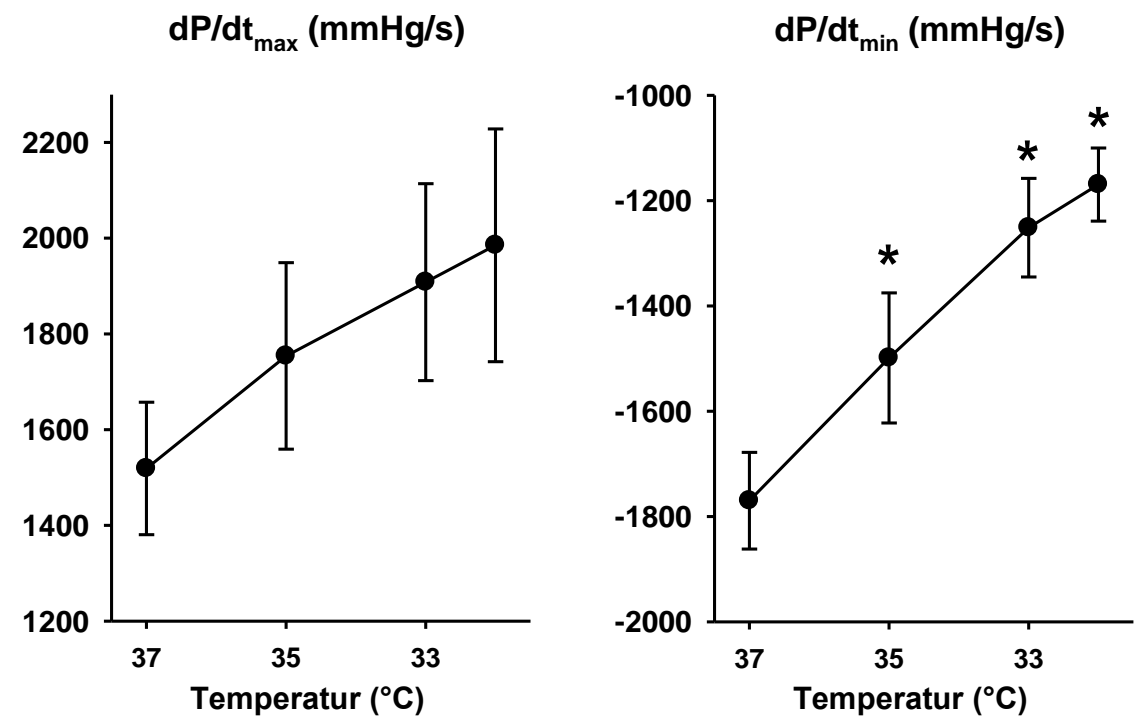

Abb. 9: $d P / d t_{\max }$ : maximale linksventrikuläre Druckanstiegsgeschwindigkeit; $d P / d t_{\min }:$ maximale Druckabfallsgeschwindigkeit 
Das $\mathrm{dP} / \mathrm{dt}_{\max }$ nahm während Hypothermie tendenziell, aber nicht signifikant $\mathrm{zu}(\mathrm{P}=0.09)$. Das $d P / d t_{\min }$ hingegen verringerte sich wie das $\mathrm{T}$ bereits bei $35^{\circ} \mathrm{C}$ deutlich; somit ist die aktive Relaxation bereits bei gering ausgeprägter Abkühlung relevant verlangsamt (Abb.9).
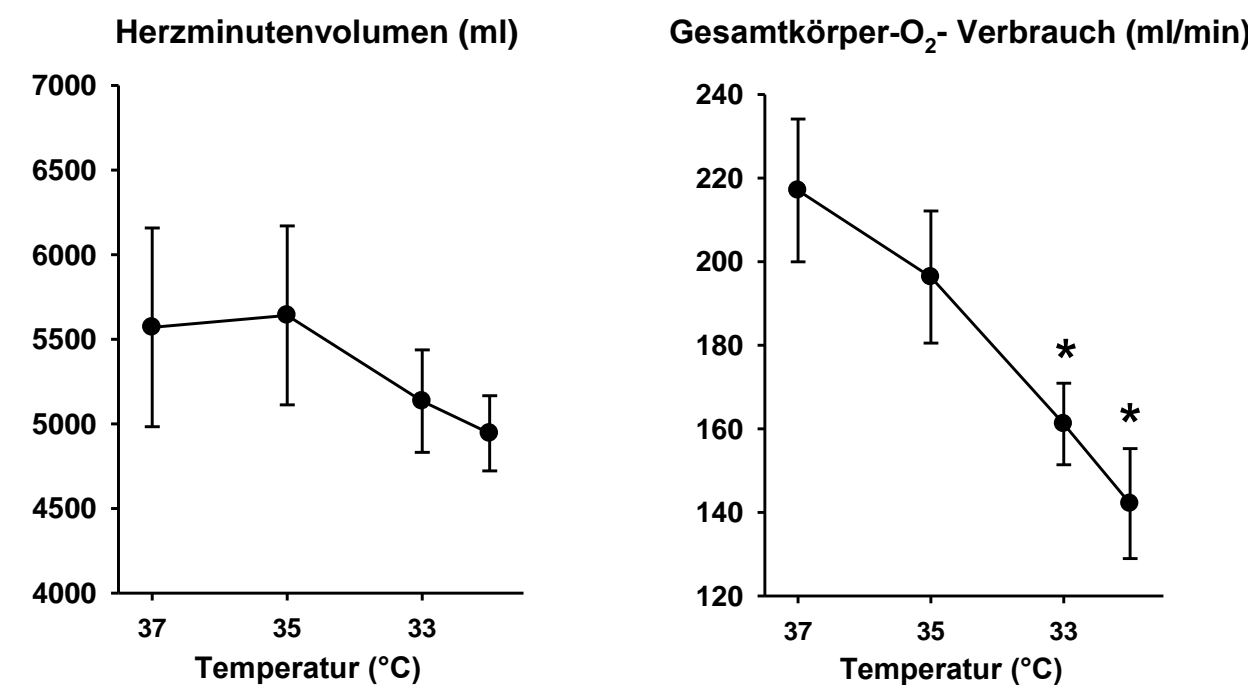

Abb. 10: mit sinkender Temperatur tendenzieller Abfall des HMV und signifikante Reduktion des Gesamtkörper - $\mathrm{O}_{2}$ - Verbrauchs

Enddiastolische, endsystolische und Schlag-Volumina änderten sich während Hypothermie nur geringfügig (Tabelle 2). Entsprechend nahm das HMV mit sinkender Herzfrequenz tendenziell, aber nicht signifikant ab (Abb.10).

Die Sauerstoffsättigung im aortalen Blut blieb konstant bei $\geq 99 \%$. Im pulmonalarteriellen Blut nahm die Sauerstoffsättigung während Hypothermie zu (Tabelle 2) und die Gesamtkörper-Sauerstoffausschöpfung damit ab. Daher sank auch der Gesamtkörpersauerstoffverbrauch während Hypothermie um ca. $6 \%$ pro $1 \mathrm{Grad}^{\circ} \mathrm{C}$ ab (Abb. 10). 
Blut - pH, Natrium-, Kalium-, Glukose-, und Laktatkonzentrationen lagen während der gesamten Dauer des Versuches sowohl aortal als auch pulmonalarteriell im Normbereich und änderten sich nicht. Mit der fixen Volumengabe nahm der Hämoglobingehalt von $10.9 \pm 0.3 \mathrm{~g} / \mathrm{dl}$ bei $37^{\circ} \mathrm{C}$ auf $10.2 \pm 0.3 \mathrm{~g} / \mathrm{dl}$ bei $35^{\circ} \mathrm{C}, 9.9 \pm 0.4 \mathrm{~g} / \mathrm{dl}$ bei $33^{\circ} \mathrm{C}\left(\mathrm{p}<0.05 \mathrm{vs} .37^{\circ} \mathrm{C}\right.$ ) und $9.6 \pm 0.4$ $\mathrm{g} / \mathrm{dl}$ bei $32^{\circ} \mathrm{C}\left(\mathrm{p}<0.05\right.$ vs. $\left.37^{\circ} \mathrm{C}\right)$ ab.

Tabelle 2: Änderung während Hypothermie bei spontaner Herzfrequenz.

$t_{s y s}(m s)$ : absolute Dauer der Systole; $t_{\text {dia }}$ (ms): absolute Dauer der Diastole; $L V D_{\text {ed: }}$ : LV end-diastolischer Druck; $V_{\text {ed }}$ : end-diastolisches LV Volumen; $V_{e s}$ : LV endsystolisches Volumen; SV: Schlagvolumen; ven. $\mathrm{O}_{2}$-Sät: pulmonalarterielle Sauerstoffsättigung; *: $p<0.05$ vs $37^{\circ} \mathrm{C}$

\begin{tabular}{|c|c|c|c|c|c|c|c|c|c|c|c|c|}
\hline & $37^{\circ} \mathrm{C}$ & & & $35^{\circ} \mathrm{C}$ & & & $33^{\circ} \mathrm{C}$ & & & $32^{\circ} \mathrm{C}$ & & \\
\hline $\mathrm{t}_{\text {sys }}(\mathrm{ms})$ & 338 & \pm 7 & & 395 & \pm & & 437 & \pm & $14^{*}$ & 464 & \pm & $21^{*}$ \\
\hline $\mathrm{t}_{\text {dia }}(\mathrm{ms})$ & 396 & \pm 3 & 35 & 394 & \pm & 35 & 392 & \pm & 34 & 383 & \pm & 35 \\
\hline $\begin{array}{l}\mathrm{LVD}_{\mathrm{ed}} \\
(\mathrm{mmHg})\end{array}$ & 9 & \pm 1 & & 11 & \pm & 1 & 12 & \pm & 1 & 13 & \pm & $1^{*}$ \\
\hline $\mathrm{V}_{\mathrm{ed}}(\mathrm{ml})$ & 138 & \pm 1 & 13 & 138 & \pm & 12 & 129 & \pm & 11 & 134 & \pm & 12 \\
\hline $\mathrm{V}_{\mathrm{es}}(\mathrm{ml})$ & 69 & \pm 7 & 7 & 64 & \pm & 9 & 59 & \pm & 8 & 65 & \pm & 10 \\
\hline $\mathrm{SV}(\mathrm{ml})$ & 69 & \pm & 9 & 73 & \pm & 7 & 70 & \pm & 5 & 70 & \pm & 5 \\
\hline $\begin{array}{l}\text { ven. } \mathrm{O}_{2} \text {-Sät } \\
(\%)\end{array}$ & 73.3 & \pm 1 & 1.8 & 76.7 & \pm & 2.3 & 79.5 & \pm & $1.6^{*}$ & 80.2 & \pm & $1.3^{*}$ \\
\hline
\end{tabular}




\subsection{Linksventrikuläre Funktion und systemische Hämodynamik unter Herzfrequenzsteigerung}

Bei $37^{\circ} \mathrm{C}$ und $33^{\circ} \mathrm{C}$ wurde rechtsatrial mit $100 /$ min und weiter mit $125 / \mathrm{min}$, 150/min und 175/min stimuliert, solange einer jeden Stimulation eine mechanische Kontraktion folgte. Die bei allen Tieren maximal erzielbare Herzfrequenz lag für $37^{\circ} \mathrm{C}$ bei $150 / \mathrm{min}$, für $35^{\circ} \mathrm{C}$ und $33^{\circ} \mathrm{C}$ bei $125 / \mathrm{min}$, und für $32^{\circ} \mathrm{C}$ bei $100 / \mathrm{min}$.
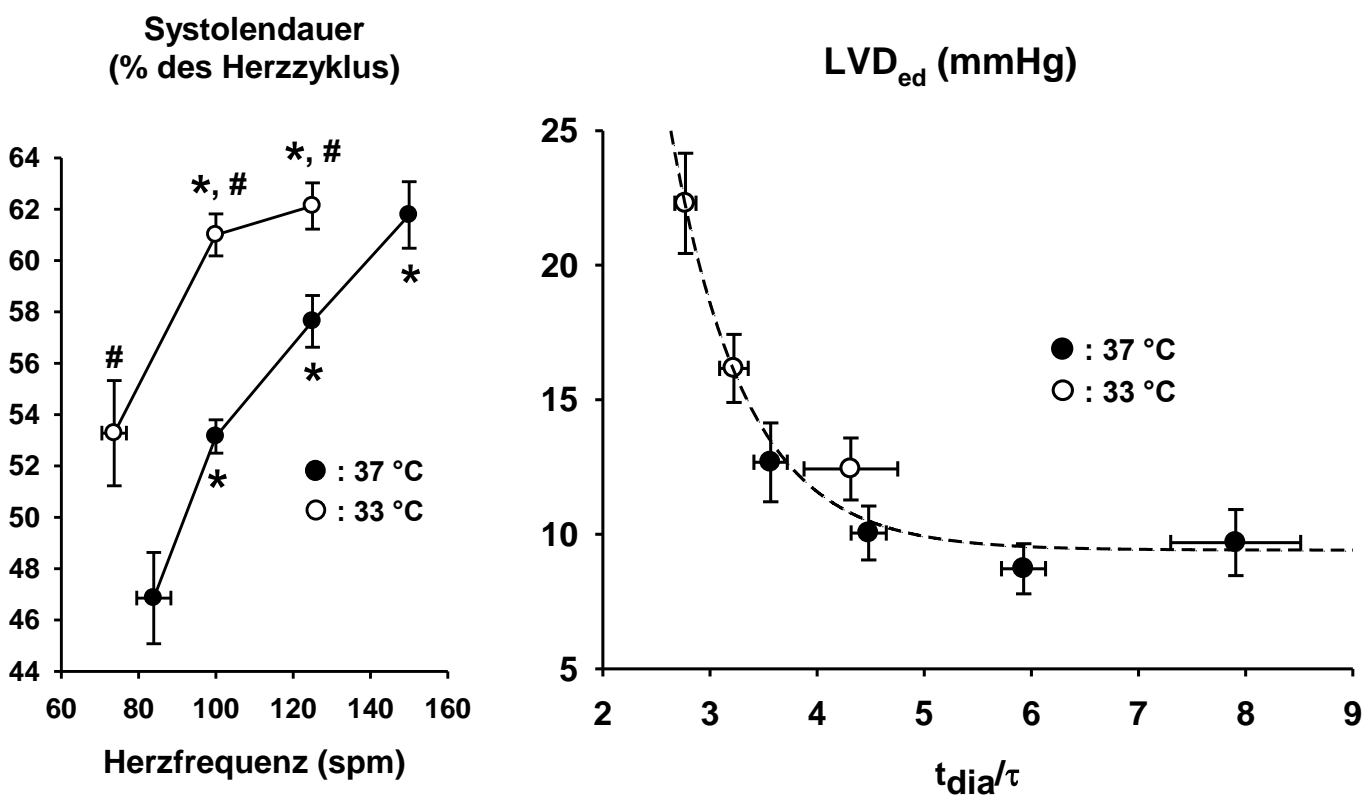

Abb. 11: links: Zunahme der relativen Systolendauer mit steigender Herzfrequenz und sinkender Temperatur; rechts: Zunahme des $L V D_{\text {ed }}$ bei einem $t_{d i a} / T<3.5$ und bei $33^{\circ} \mathrm{C}$

Die Schrittmacherstimulation verursachte eine Verkürzung der absoluten und relativen Diastolendauer (Tabelle 2), dieser Effekt war bei $33^{\circ} \mathrm{C}$ potenziert. Der enddiastolische Druck nahm bei $37^{\circ} \mathrm{C}$ etwas zu, während das enddiastolische Volumen abnahm; auch dieser Effekt war bei $33^{\circ} \mathrm{C}$ verstärkt. 
Der $L V D_{\text {es }}$ nahm bei den höchsten Stimulationsraten ab. Das $\mathrm{dP} / \mathrm{dt}_{\max }$ blieb während Schrittmacherstimulation bei $37^{\circ} \mathrm{C}$ konstant und nahm bei Hypothermie ab. Das $\mathrm{dP} / \mathrm{dt}_{\min }$ blieb bei $33^{\circ} \mathrm{C}$ versus $37^{\circ} \mathrm{C}$ vermindert. Das Schlagvolumen nahm bei beiden Temperaturen während Stimulation ab.

Der Anstieg des enddiastolischen Drucks konnte abgeleitet werden vom Verhältnis der absoluten Diastolendauer $\left(t_{\text {dia }}\right)$ zur Zeitkonstante der isovolumetrischen Relaxation ( $\mathrm{T}$ ) mit Hilfe der folgenden exponentiellen Gleichung:

$L V D_{\text {ed }}=C+A \cdot \exp \left(-B .\left(t_{\text {dia }} / \square\right)\right)$

Eine Konstante wurde berücksichtigt, da der enddiastolische Druck bei positivem endexspiratorischem Druck keine Nullwerte annehmen konnte. Die berechneten Parameter der Kurvenanpassung lauteten wie folgt:

$C=9.4 \pm 0.7 \mathrm{mmHg} ; A=701 \pm 599 \mathrm{mmHg} ; B=1.4 \pm 0.3 ; r^{2}=0.95 ; p=0.001$. 
Tabelle 3: $t_{\text {sys: }}$ : absolute Systolendauer, $t_{\text {dia }}$ : absolute Diastolendauer, $L V D_{e s}$ : endsystolischer Druck, $d P / d t_{\max }$ : maximale Druckanstiegsgeschwindigkeit, $d P / d t_{\min }$ : maximale Druckabfallgeschwindigkeit, $r$ : Zeitkonstante der isovolumetrischen Relaxation, $V_{e d:}$ enddiastolisches Volumen, SV: Schlagvolumen

\begin{tabular}{|c|c|c|c|c|c|c|c|c|c|}
\hline & & sp & & $100 / \mathrm{mi}$ & & $125 / \mathrm{mi}$ & & $150 / \mathrm{mi}$ & \\
\hline \multirow{2}{*}{$\mathrm{t}_{\text {sys }}(\mathrm{ms})$} & $37^{\circ} \mathrm{C}$ & 338 & \pm 7 & 311 & $\pm 4^{*}$ & 274 & $\pm 5^{*}$ & 244 & $\pm 5^{*}$ \\
\hline & $33^{\circ} \mathrm{C}$ & 437 & $\pm \quad 14$ & 366 & $\pm 5^{\star}, \#$ & 297 & $\pm 5^{*}, \#$ & & \\
\hline \multirow{2}{*}{$\mathrm{t}_{\text {dia }}(\mathrm{ms})$} & $37^{\circ} \mathrm{C}$ & 396 & \pm 35 & 275 & $\pm 5^{*}$ & 201 & $\pm 5^{*}$ & 151 & $\pm 5^{*}$ \\
\hline & $33^{\circ} \mathrm{C}$ & 392 & $\pm \quad 34$ & 226 & $\pm 6^{*}, \#$ & 181 & $\pm 6^{*}$ & & \\
\hline \multirow{2}{*}{$\begin{array}{l}\mathrm{LVD}_{\mathrm{es}} \\
(\mathrm{mmHg})\end{array}$} & $37^{\circ} \mathrm{C}$ & 111 & \pm 4 & 112 & \pm 5 & 109 & \pm 4 & 101 & $\pm 4^{*}$ \\
\hline & $33^{\circ} \mathrm{C}$ & 125 & \pm 8 & 123 & \pm 8 & 112 & $\pm 9^{*}$ & & \\
\hline \multirow{2}{*}{$\begin{array}{l}\mathrm{dP} / \mathrm{dt}_{\max } \\
(\mathrm{mmHg} / \mathrm{s})\end{array}$} & $37^{\circ} \mathrm{C}$ & 1519 & \pm 138 & 1485 & \pm 121 & 1499 & \pm 98 & 1491 & \pm 100 \\
\hline & $3^{\circ} \mathrm{C}$ & 1908 & \pm 206 & 1850 & \pm 185 & 1735 & $\pm 191^{*}$ & & \\
\hline \multirow{2}{*}{$\begin{array}{l}\mathrm{dP} / \mathrm{dt}_{\min } \\
(\mathrm{mmHg} / \mathrm{s})\end{array}$} & $37^{\circ} \mathrm{C}$ & 1770 & \pm 92 & 1868 & \pm 86 & 1861 & \pm 69 & 1770 & \pm 60 \\
\hline & $33^{\circ} \mathrm{C}$ & 1252 & $\pm 94 \#$ & 1486 & $\pm 83^{*}, \#$ & 1387 & $\pm 121 \#$ & & \\
\hline \multirow{2}{*}{$\square(\mathrm{ms})$} & $37^{\circ} \mathrm{C}$ & 50 & \pm 2 & 47 & $\pm 1^{*}$ & 45 & $\pm 1^{*}$ & 42 & $\pm 1^{*}$ \\
\hline & $33^{\circ} \mathrm{C}$ & 100 & \pm 15 & 71 & \pm 3 & 66 & $\pm 2^{*}, \#$ & & \\
\hline \multirow{2}{*}{$\mathrm{V}_{\text {ed }}(\mathrm{ml})$} & $37^{\circ} \mathrm{C}$ & 138 & \pm 13 & 130 & \pm 13 & 115 & \pm 11 & 97 & $\pm 10^{*}$ \\
\hline & $33^{\circ} \mathrm{C}$ & 129 & \pm 11 & 111 & $\pm 10 \#$ & 86 & $\pm 9^{*}, \#$ & & \\
\hline \multirow{2}{*}{$\mathrm{SV}(\mathrm{ml})$} & $37^{\circ} \mathrm{C}$ & 69 & \pm 9 & 58 & $\pm 7^{*}$ & 45 & $\pm 5^{*}$ & 34 & $\pm 4^{*}$ \\
\hline & $33^{\circ} \mathrm{C}$ & 70 & \pm 5 & & $\pm 4^{*}$ & & $\pm 4^{*}$ & & \\
\hline
\end{tabular}




\subsection{Druck - Volumen - Beziehungen}

Der primäre Effekt der Hypothermie auf die Position der linksventrikulären Druck - Volumenbeziehung war eine Verschiebung nach links und oben, so dass ein gegebener endsystolischer oder enddiastolischer Druck bereits bei geringeren Volumina erreicht wurde (s. Abb. 12).

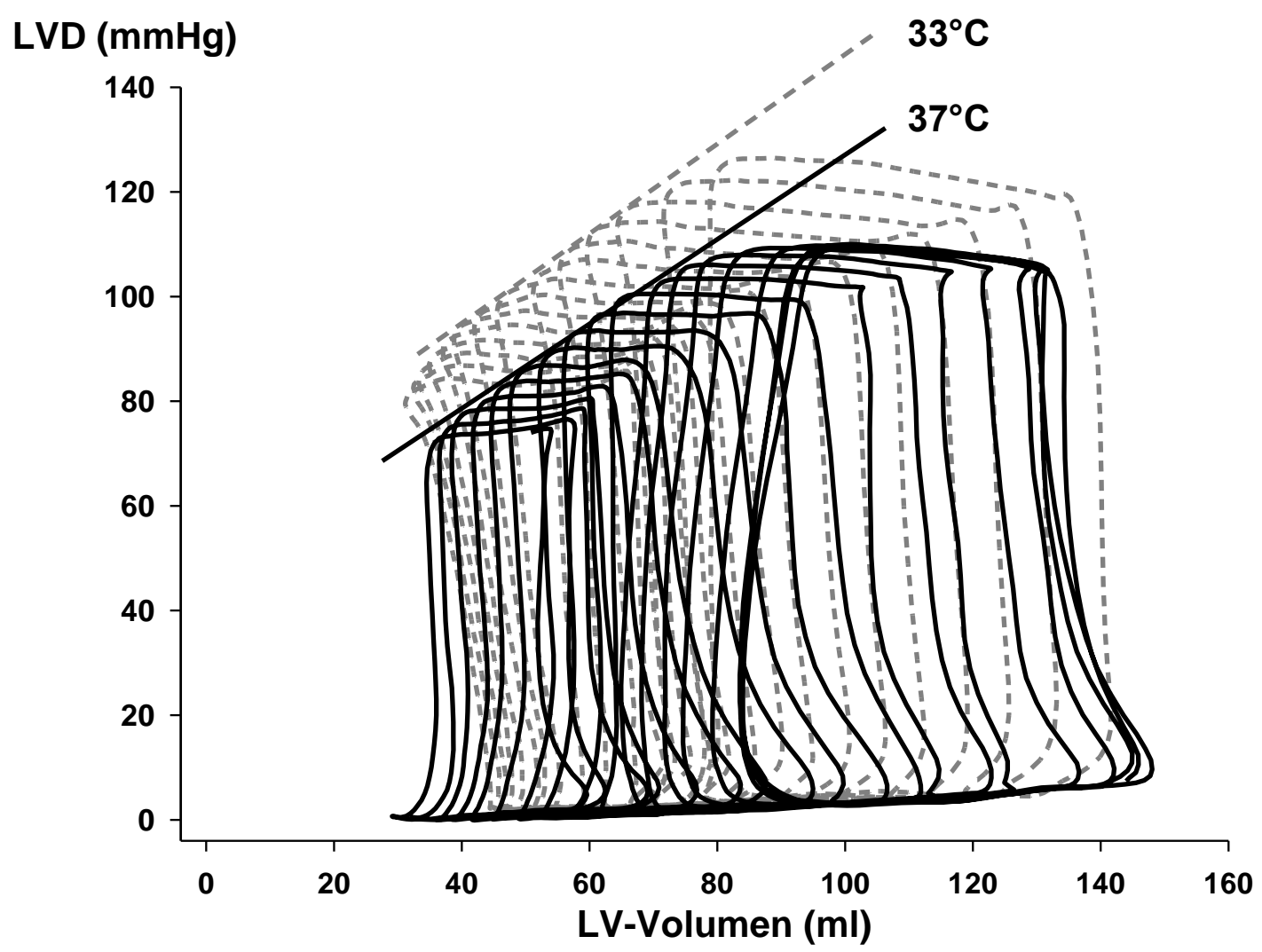

Abb. 12: Druckvolumenschleifen des linken Ventrikels während akuter Vorlastreduktion durch V.- cava- inf.- Okklusion bei Normo- und bei Hypothermie.

Die lineare Regressionsanalyse ergab keine signifikante Änderung der Steigungen von EDDVB und ESDVB. Auch bei einer Frequenz von 100/min änderte sich die Steigung von ESDVB unter Hypothermie nicht. (siehe Tab. 4) Jedoch kam es zu einer parallelen Linksverschiebung der 
Regressionsgeraden von EDDVB / ESDVB entlang der $x$-Achse. Dementsprechend sanken die errechneten Volumina für einen endsystolischen Druck von 120 mmHg unter Hypothermie. Dieser Effekt war sowohl unter spontaner Herzfrequenz als auch bei 100/min zu beobachten (siehe Abb. 13).

$$
\begin{aligned}
& \text { errechnetes Volumen }(\mathrm{ml}) \\
& \text { bei } L V D_{\text {es }}=120 \mathrm{mmHg}
\end{aligned}
$$

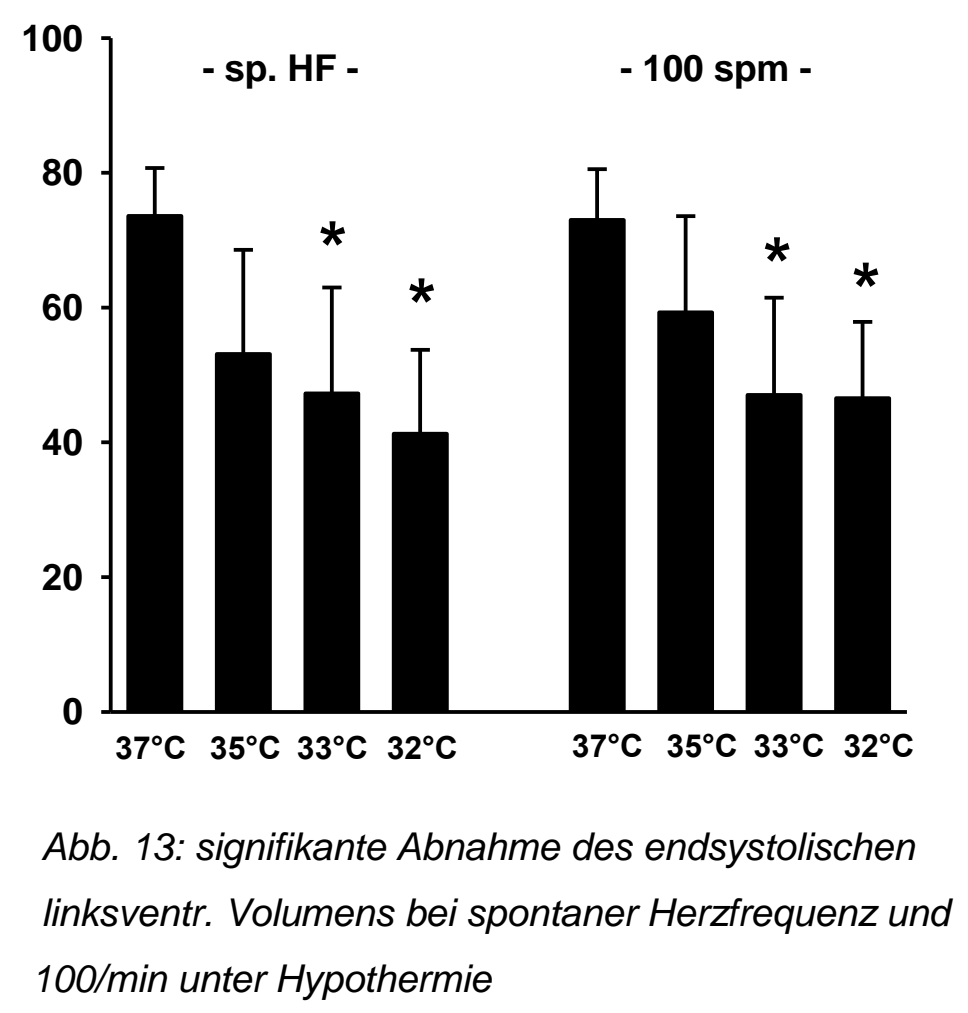

Gleichermaßen nahmen auch die errechneten Volumina für einen enddiastolischen Druck von 10 mmHg unter Hypothermie ab (siehe Abb. 14). 


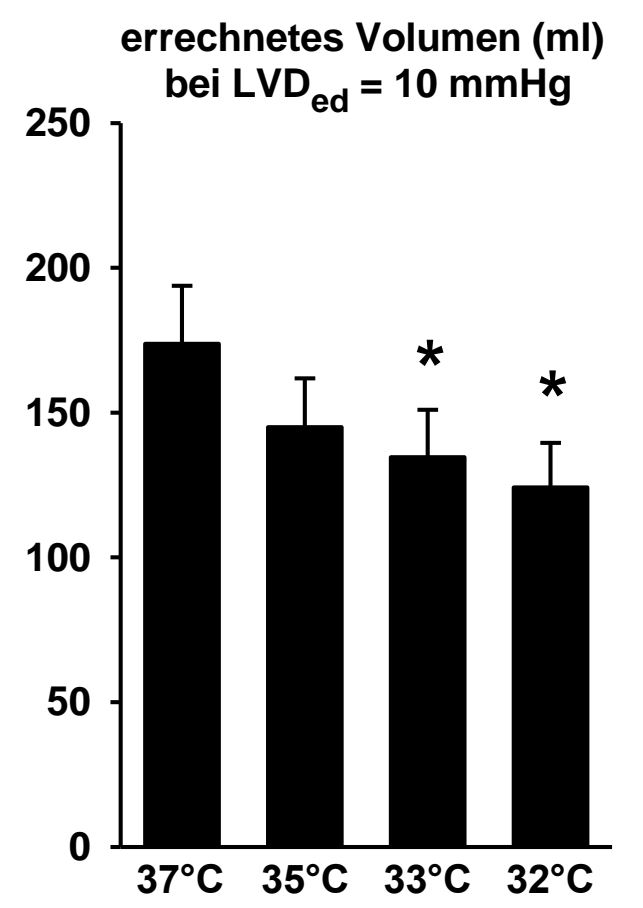

Abb. 14: signifikante Abnahme des enddiastolischen linksventr. Volumens unter Hypothermie

Tabelle4: $E D D V B_{s p}$ : enddiastolische Druck-Volumenbeziehung bei spontaner Herzfrequenz; ESDVB $B_{s p}$ : endsystolische Druck-Volumenbeziehung bei spontaner Herzfrequenz; ESDVB ${ }_{100 / m i n:}$ endsystolische Druck-Volumenbeziehung bei Herzfrequenz $=100 / \mathrm{min}$

\begin{tabular}{|c|c|c|c|c|c|c|c|c|c|c|c|c|}
\hline \multirow{2}{*}{$\begin{array}{l}\text { EDDVB }_{\mathrm{sp}} \\
(\mathrm{mmHg} / \mathrm{ml})\end{array}$} & \multicolumn{3}{|l|}{$37^{\circ} \mathrm{C}$} & \multicolumn{3}{|l|}{$35^{\circ} \mathrm{C}$} & \multicolumn{3}{|l|}{$33^{\circ} \mathrm{C}$} & \multicolumn{3}{|l|}{$32^{\circ} \mathrm{C}$} \\
\hline & 0.083 & \pm & 0.011 & 0.095 & \pm & 0.008 & 0.093 & \pm & 0.006 & 0.104 & \pm & 0.018 \\
\hline $\begin{array}{l}\text { ESDVB }_{\mathrm{sp}} \\
(\mathrm{mmHg} / \mathrm{ml})\end{array}$ & 0.89 & \pm & 0.12 & 0.90 & \pm & 0.10 & 0.86 & \pm & 0.10 & 0.92 & \pm & 0.16 \\
\hline $\begin{array}{l}\text { ESDVB }_{100 / m i n} \\
(\mathrm{mmHg} / \mathrm{ml})\end{array}$ & 0.87 & \pm & 0.13 & 0.95 & \pm & 0.13 & 0.99 & \pm & 0.13 & 1.03 & \pm & 0.18 \\
\hline
\end{tabular}




\section{Diskussion}

\subsection{Diskussion der Methode}

In der vorliegenden Studie wurde die milde Hypothermie durch einen intravasalen Kühlkatheter induziert und aufrechterhalten. Diese Methode gewährleistet anders als z.B. eine Kühlung durch Eispacks eine präzise Kontrolle der Kerntemperatur und vermeidet zudem einen Temperaturgradienten von außen nach innen, welcher ein starkes Kältezittern hervorruft. Weiterhin ist diese Methode weniger invasiv als beispielsweise eine Kühlung über eine Herz-Lungenmaschine.

Die linksventrikuläre Kontraktilität kann, wie in der Einleitung ausgeführt, nur durch Bestimmung mehrerer Parameter erfasst werden und sollte idealerweise Vor- und Nachlast sowie die Herzfrequenz berücksichtigen. Die hier angewandte Methode der hochauflösenden Messung von Druck und Volumen unter Vorlastvariation und Schrittmacherstimulation erfült diese Anforderungen.

Wir konnten dieses Modell durch Katheterverfahren ohne Eröffnung der Thoraxhöhle und mit Einsatz von in der Klinik gebräuchlichen Anästhetika realisieren, so dass die Ergebnisse nächst möglich am klinischen Szenario gewonnen wurden. So wurde auf den Einsatz kardiodepressiver Barbiturate verzichtet und auf eine ausreichende muskuläre Relaxation geachtet, um ein energieverbrauchendes Kältezittern zu vermeiden.

Kritisch anzumerken ist, dass die physiologische Körpertemperatur von Schweinen über der des Menschen bei ca. $38^{\circ} \mathrm{C}$ liegt. Jedoch war der wählbare Temperaturbereich durch das eingesetzte Gerät limitiert, welches zum Zeitpunkt der Studie den Stand dieser neuen Technik repräsentierte. 


\subsection{Diskussion der Ergebnisse}

\subsubsection{Metabolisches Gleichgewicht bei milder Hypothermie}

In der vorliegenden Arbeit sank die Herzfrequenz proportional zur Temperatur ab, während für die Parameter endsystolischer linkventrikulärer Druck, linksventrikuläre Volumina und HMV nur geringe, nicht signifikante Änderungen während Hypothermie zu verzeichnen waren. Bemerkenswerterweise nahm der Gesamtkörpersauerstoffverbrauch von $37^{\circ} \mathrm{C}$ auf $33^{\circ} \mathrm{C}$ um ca. $25 \%$ ab. In Anbetracht der tiefen Narkose einschließlich kompletter muskulärer Relaxation ist der geringere Sauerstoffverbrauch als eindeutiger Effekt der $\mathrm{MH}$ anzusehen. Der Anstieg der zentral-venösen Sättigung und die Stabilität anderer metabolischer Parameter wie $\mathrm{pH}-$ Wert und arterielle Laktatkonzentration zeigen, dass der Gesamtkörperenergiebedarf in dieser Versuchsreihe ausreichend gedeckt war. Es ist somit vorstellbar, dass der moderate Abfall des HMV unter MH in vorangegangenen Studien (Boddicker et al. 2005; Fischer et al. 2005; Perezde-Sa et al. 2002; Tveita et al. 1998) kein Anzeichen einer verminderten Sauerstoffversorgung darstellte, sondern eher auf einen reduzierten Energiebedarf des Körpers schließen lässt, der dementsprechend durch ein geringeres HMV gedeckt wurde. 


\subsubsection{Kardiale Inotropie während MH}

Weisser et al. (2001) konnten in ihrer Studie an isolierten Herzmuskelstreifen von Schweinen anhand verlängerter Zeiten bis zur maximalen Kraftentwicklung (TTP) sowie prolongierten Relaxationszeiten ( $\left.R T_{50}\right)$ zeigen, dass der Zuwachs an Zuckungskraft während Hypothermie charakteristischerweise mit einer Verlängerung des Herzzyklus einhergeht. Einhergehend mit der beschriebenen Verlängerung der Kontraktion konnten Weisser et al. weiterhin zeigen, dass sich die Kraftentwicklungsgeschwindigkeit trotz gesteigerter maximal erreichter Kraft nicht ändert. Dieser Effekt der MH steht im Gegensatz zum positiv inotropen Effekt von beta-Adrenorezeptoragonisten, welche sowohl die maximale Kraft als auch die Kontraktionsgeschwindigkeit steigern. Jener Gegensatz zwischen gesteigerter Kontraktionskraft und gleich bleibender Kontraktionsgeschwindigeit ist gleichermaßen an isolierten Herzen beschrieben worden, bei denen zwar für die Steilheit der endsystolischen Druck-Volumenbeziehung, jedoch nicht für die maximale Druckanstiegsgeschwindigkeit eine Steigerung während $\mathrm{MH}$ zu verzeichnen war (Fukunami und Hearse 1989). Auch in der hier vorliegenden Arbeit verlängerte sich die Systolendauer unter Hypothermie. Im Gegensatz zu den zuvor zitierten Arbeiten konnten wir eine Steigerung der maximalen Druckanstiegsgeschwindigkeit zeigen, welche jedoch unterhalb des Signifikanzniveaus ( $p=0.09$ ) lag. Diese Ergebnisse erscheinen in Anbetracht der verlangsamten Kontraktionsvorgänge unter Hypothermie plausibel.

Bei der Betrachtung der Steigung der endsystolischen DruckVolumenbeziehung, die als in-vivo-Äquivalent der isometrischen Kraftentwicklung im isolierten Muskelstreifen betrachtet werden kann, ergaben sich in der hier vorliegenden Arbeit keine signifikanten Änderungen. Jedoch nahmen die errechneten Volumina für einen Druck von $120 \mathrm{mmHg}$ 
mit sinkenden Temperaturen stetig ab. Nach Burkhoff et al. (2005) dürfen jedoch bei in-vivo-Versuchen weder der y - Achsenabschnitt noch der Anstieg der ESDVB separat betrachtet werden. Vielmehr sollte die veränderte Position der ESDVB im Ganzen und im Bereich physiologischer Druckwerte beurteilt werden um ein relevantes Maß für Inotropie zu erhalten. Daher kann die parallele Linksverschiebung der ESDVB in dieser Arbeit tatsächlich als gesteigerte Inotropie gedeutet werden, was mit Ergebnissen an Hundeherzen in vitro (Suga et al. 1988) und in vivo (Nishimura et al. 2005) übereinstimmt. Bei einer Studie, die an Patienten durchgeführt wurde, welche sich einer Bypass-Operation unterzogen, stellte sich interessanterweise heraus, dass die Steigung der ESDVB unter Hypothermie abnahm, was einen negativ inotropen Effekt der MH implizieren würde (Lewis et al. 2002). Der tatsächlich gemessene endsystolische Druck von beispielsweise $90 \mathrm{mmHg}$ wurde dahingegen bei geringeren anstatt höheren endsystolischen Volumina erreicht, was wiederum für eine gesteigerte Inotropie spricht.

Somit zeigt sich der positiv inotrope Effekt der Hypothermie offenbar nicht in einer Beschleunigung der Kontraktion (fast unverändertes $\mathrm{dP} / \mathrm{dt}_{\max }$ ) jedoch in einer erhöhten Kontraktionskraft (Linksverschiebung der ESDVB). 


\subsubsection{Diastolische Funktion während MH}

In der hier vorliegenden Arbeit konnte gezeigt werden, dass die Induktion der MH die diastolische Funktion des linken Ventrikels in mehrerer Hinsicht beeinträchtigt.

Erstens deuten die signifikanten Veränderungen in $\mathrm{dP} / \mathrm{dt}_{\min }$ und $\mathrm{T}$ auf eine Verlangsamung der aktiven linksventrikulären Relaxation hin, was schon bei einer geringen Temperaturänderung von $37^{\circ} \mathrm{C}$ auf $35^{\circ} \mathrm{C}$ zu beobachten war. Zweitens blieb, anders als bei der Ruhebradykardie unter Normothermie, die Diastolendauer unter Hypothermie - induzierter Bradykardie konstant, während sich die Systolendauer verlängerte. Weiterhin ließ sich eine Linksverschiebung der enddiastolischen Druck - Volumenbeziehung (EDDVB) erkennen, was den dritten Aspekt der diastolischen Funktionsstörung unter $\mathrm{MH}$ kennzeichnet.

Während die Beeinträchtigung der Relaxation als Effekt der $\mathrm{MH}$ bereits häufig beschrieben worden ist (Boyer und Gerstein 1977), konnte eine Linksverschiebung der EDDVB in vivo bisher noch nicht gezeigt werden. Die Ursache dieser Verschiebung könnte in einer Hypothermie - induzierten erhöhten linksventrikulären Steifheit oder, beziehungsweise und in einer vermehrten Beeinträchtigung der aktiven Relaxation unter $\mathrm{MH}$ liegen. Um diese Frage beantworten zu können erhöhten wir die Herzfrequenz mittels rechtsatrialer Schrittmacherstimulation. Die Herzfrequenzerhöhung führte zu einer relativen Verlängerung der Systole und zur Erhöhung der enddiastolischen linksventrikulären Drücke. Dieser Effekt verstärkte sich unter $\mathrm{MH}$. Unter der Annahme, dass die aktive Relaxation bei $97 \%$ des linksventrikulären Druckabfalls abgeschlossen ist, kann die Diastolendauer, die dafür notwendig ist, entsprechend des exponentiellen Verlaufes von $\mathrm{T}$, aus 3.5 x T errechnet werden (Hay et al. 2005; Leite-Moreira und Gillebert 2000). Wir stellten daher die linksventrikulären enddiastolischen Drücke bei 
spontaner und stimulierter Herzfrequenz als Funktion des Verhältnisses von Diastolendauer und $\mathrm{T}$ dar und konnten tatsächlich eine enge Beziehung mit deutlich ansteigenden enddiastolischen linksventrikulären Drücken bei einem Verhältnis $<3.5$ beobachten. Diese Ergebnisse zeigen, dass die Fähigkeit des linken Ventrikels zur aktiven Relaxation während $\mathrm{MH}$, insbesondere bei erhöhten Herzfrequenzen, stark beeinträchtigt ist und maßgeblich zu den beobachteten erhöhten enddiastolischen Drücken während $\mathrm{MH}$ beiträgt. Ferner bestätigten diese Resultate die Computersimulation von Hay et al. (2005), welche annehmen, dass die Verlängerung der Systole und die Beeinträchtigung der Relaxation, insbesondere bei höheren Herzfrequenzen, zu erhöhten enddiastolischen Drücken beitragen kann. Jedoch gingen in der hier vorliegenden Arbeit die erhöhten linksventrikulären enddiastolischen Drücke mit signifikant verringerten enddiastolschen Volumina einher; dies geht über das Modell von Hay et al. hinaus. 


\subsubsection{Potentielle subzelluläre Mechanismen der milden Hypothermie}

Auf der Ebene des Kardiomyozyten ist die Kontraktion des Herzens durch eine, an einen elektrischen Stimulus gekoppelte, phasische Freisetzung von Kalzium aus dem sarkoplasmatischen Retikulum bedingt. Katecholamine beschleunigen und erhöhen typischerweise diesen Kalziumtransienten und steigern dadurch die Anzahl der sich bildenden Quervernetzungen der Aktinund Myosinfilamente, die für die Kraftentwicklung notwendig sind. Der Effekt der milden Hypothermie liegt hingegen in einer Verzögerung ohne Änderung der Amplitude des Kalziumtransienten, was Experimente an isolierten Herzmuskelstreifen von Ratten (Hiranandani et al. 2006) und Schweinen (Weisser et al. 2001) ergaben. Ebenfalls war bei Weisser et al. der Gehalt des sarkoplasmatischen Retikulums an Kalzium während $\mathrm{MH}$ nicht erhöht. Suga et al. schlussfolgerten, dass "[...] der erhöhte Sauerstoffverbrauch ... unter Epinephrin oder Kalzium in erster Linie durch einen gesteigerten Energieverbrauch in Verbindung mit der elektromechanischen Kopplung verursacht ist." (Suga et al. 1983, S. 306). Eine Erhöhung der Kontraktilität durch milde Hypothermie anstatt mittels Katecholaminen ist somit energieeffizienter als z.B. durch Epinephrin, da nur wenig zusätzliche Energie für die intrazellulären Kalziumbewegungen benötigt wird (Suga et al. 1983).

Wenn der Kaziumtransient unverändert bleibt, muss die Ursache der kontraktilitätssteigernden Wirkung der Hypothermie in einer veränderten Antwort der Myofilamente auf das Kalzium liegen. Ein solcher Effekt könnte daher entweder auf einer gesteigerten Kalziumsensitivität der Myofilamente oder einer Zunahme der Kalzium - induzierten Kraft beruhen (Lee und Allen 1997). 
Eine Erhöhung der Kalziumsensitivität der Myofilamente konnte im Rahmen der milden Hypothermie jedoch nicht beobachtet werden (Nakae et al. 2001). Auch die Theorie einer intrazellulären Alkalose, welche wiederum die Kalziumsensitivität erhöhen würde, konnte in Experimenten an isolierten Herzen für einen Temperaturbereich von $37^{\circ} \mathrm{C}$ bis $32^{\circ} \mathrm{C}$ widerlegt werden (Kusuoka et al. 1991). Jedoch konnte in der vorangegangenen Studie demonstriert werden, dass mit Induktion der MH die Kalzium - abhängige Kraft zunimmt (Kusuoka et al. 1991). Die Ursache hierfür könnte eine Verlangsamung der Quervernetzungsvorgänge zwischen den Aktin- und Myosinfilamenten sein (Henderson und Cattell 1976), was eine plausible Erklärung für die verlängerte Kontraktion wäre. Im Zusammenhang mit einer Verlangsamung der temperaturabhängigen sarkoplasmatischen Kalzium ATPase (Inesi und Watanabe 1967) könnte der zuvor beschriebene Effekt zudem zu der Verlängerung des Kalziumtransienten und der verzögerten Relaxation beitragen.

In der vorliegenden Arbeit konnte eine Linksverschiebung der EDDVB während Hypothermie demonstriert werden. Dieser Effekt konnte zuvor in Experimenten an isolierten Herzen beobachtet werden (Remensnyder und Austen 1965; Templeton et al. 1974) und wurde mit einer Steigerung der Viskosität des Herzmuskels unter MH erklärt (Templeton et al. 1974). Ferner wurden Veränderung des Phosphorylierungszustandes von Strukturproteinen des Herzmuskels, beispielsweise Titin, im Zusammenhang mit einer beeinträchtigten EDDVB beschrieben (Kass et al. 2004), was bisher jedoch nicht unter Bedingungen der $\mathrm{MH}$ untersucht worden ist. 


\subsection{Schlussfolgerung und klinische}

\section{Anwendbarkeit}

Im Rahmen einer kardiopulmonalen Wiederbelebung kommt es zu einer postischämischen Dysfunktion des Herzens, so dass es häufig erforderlich ist, die Kontraktilität durch Applikation positiv inotroper Pharmaka aufrecht zu erhalten. Alternativ könnte der Einsatz der milden Hypothermie und die daraus resultierende Steigerung der Inotropie als Therapieoption gegen dieses so genannte Stunning in Erwägung gezogen werden, wobei dann jedoch eine simultane Verschlechterung der diastolischen Herzfunktion beobachtet werden kann. Der routinemäßige Einsatz der $\mathrm{MH}$ bei Patienten mit stabilem Kreislauf zeigt jedoch, dass diese diastolische Funktionsstörung durch die Hypothermie - induzierte Bradykardie ausgeglichen werden kann. Dahingegen kann sich bei Patienten mit linksventrikulärer Hypertrophie und präexistenter diastolischer Funktionsstörung oder mit kritischer Koronarstenose, bei der die Verkürzung der Diastole und die erhöhten linksventrikulären diastolischen Drücke die Koronarperfusion noch weiter einschränken würden, die diastolische Funktion zunehmend verschlechtern. Weiterhin könnte die Linksverschiebung der Hämoglobin Sauerstoffbindungskurve die poststenotische Sauerstoffversorgung des Myokards bei KHK - Patienten zusätzlich einschränken. Ob Hypothermie die Herzfunktion während des vermehrten Stunning nach kardiopulmonaler Wiederbelebung oder während des kardiogenen Schocks verbessert, ist weder experimentell noch klinisch im Detail untersucht. Die im Rahmen dieser Arbeit erhobenen Daten zeigen jedoch, dass sich der Einsatz der $\mathrm{MH}$ als Therapieoption bei akuter Linksherzinsuffizienz oder im kardiogenen Schock als vorteilhaft erweisen könnte.

In Studien an anästhesierten Hunden, bei denen durch Koronarverschluss eine Schocksituation mit akuter Herzinsuffizienz induziert wurde, konnte eine 
verlängerte Überlebenszeit und zu einem gewissen Grade eine verbesserte Herzfunktion unter Hypothermie belegt werden (Kuhn et al. 1963). Auch in zwei beobachtenden klinischen Studien an Kindern (Dalrymple-Hay et al. 1999) und Erwachsenen (Yahagi et al. 1998) mit beeinträchtigter Herzfunktion nach Herzoperation erwies sich der Effekt der milden Hypothermie als potentiell vorteilhaft. Des weiteren konnte in einer vorläufigen Studie gezeigt werden, dass durch den Einsatz der $\mathrm{MH}$ bei Patienten im kardiogenen Schock tatsächlich das HMV gesteigert werden konnte, wobei keinerlei nachteilige Nebeneffekte zu verzeichnen waren (Schmidt-Schweda 2004).

Einhergehend mit den zitierten Ergebnissen zeigen auch die in dieser Arbeit erhobenen Daten, dass sich der Einsatz der MH im kardiogenen Schock als vorteilhaft erweisen könnte, da zum einen der Gesamtkörpersauerstoffverbrauch beträchtlich gesenkt wird und zum anderen die kardiale Inotropie gesteigert wird. Weiterhin konnten wir zeigen, dass zwar die diastolische Funktion durch eine Verkürzung der Diastolendauer, eine verzögerte aktive Relaxation und eine verminderte enddiastolische Dehnbarkeit beeinträchtigt ist, was jedoch durch einen parallelen Abfall der spontanen Herzfrequenz kompensiert wird.

Die gesteigerte Inotropie und der verminderte Sauerstoffverbrauch unter Hypothermie geben Anlass zu weiteren experimentellen und klinischen Studien, insbesondere zur Eruierung des Nutzens der $\mathrm{MH}$ unter Bedingungen des kardiogenen Schocks. 


\section{Zusammenfassung}

Während die Induktion der milden Hypothermie zur Minderung hypoxischer Hirnschäden nach kardiopulmonaler Reanimation allgemein akzeptierter therapeutischer Standard geworden ist, werden die kardialen Effekte der Hypothermie kontrovers diskutiert. Ein positiv inotroper Effekt der Hypothermie konnte durch zahlreiche Arbeitsgruppen in in-vitro-Modellen nachgewiesen werden, wohingegen in in-vivo-Modellen widersprüchliche Effekte der Hypothermie berichtet worden sind.

10 weibliche Schweine wurden anästhesiert und mit einem Konduktanzkatheter, einem Swan-Ganz-Katheter und einem rechtsatrialen Schrittmacher katheterisiert. Über die Femoralvenen wurden ein Vena-cavaOkklusions-Ballon sowie ein Kühlkatheter zur Induktion der $\mathrm{MH}$ eingeführt. Nach Einleitung der $\mathrm{MH}$ (von $37^{\circ} \mathrm{C}$ auf $33^{\circ} \mathrm{C}$ ) sanken die Herzfrequenz $(85 \pm 4$ vs $74 \pm 3$ spm) und der Gesamtkörpersauerstoffverbrauch ( $217 \pm 17$ vs $161 \pm 10$ $\mathrm{ml} / \mathrm{min}$ ), während die arterielle Laktatkonzentration konstant blieb. Änderungen von Herzminutenvolumen, enddiastolischem Volumen, Schlagvolumen, systolischem Spitzendruck, enddiastolischem Druck und $\mathrm{dP} / \mathrm{dt}_{\max }$ lagen nicht im signifikanten Bereich. Verminderungen der maximalen Druckabfallsgeschwindigkeiten und die entsprechenden Erhöhungen der Zeitkonstante der isovolumetrischen Relaxation t zeigten eine Beeinträchtigung der aktiven linksventrikulären Relaxation. Des Weiteren nahm der Anteil der Systole am Herzzyklus zu. Die während transienter Vena-cava-Okklusion erhobenen Daten wurden zur Generierung von Druck-Volumen-Beziehungen benutzt. Die Analyse der DruckVolumendaten zeigte eine erhöhte endsystolische sowie enddiastolische Steifheit, was zum einen positive Inotropie, zum anderen eine reduzierte enddiastolische Dehnbarkeit belegt. Die positive Inotropie blieb auch bei 
Steigerung der Herzfrequenz auf 100/min erhalten, während sich die diastolische Füllung weiter verschlechterte.

Schlussfolgerung: Die Induktion der milden Hypothermie ist positiv inotrop und vermindert den Gesamtkörper- $\mathrm{O}_{2}$-Verbrauch. Die diastolische Funktion verschlechtert sich, was jedoch durch einen parallelen Abfall der spontanen Herzfrequenz kompensiert wird. Diese Daten weisen darauf hin, dass die Induktion der milden Hypothermie eine neue Behandlungsoption beim therapierefraktären akuten Herzversagen sein könnte. 


\section{Literaturverzeichnis}

Almond GW (1996): Research Applications Using Pigs. Vet Clin North Am Food Anim Pract 12: 707-716.

Andresen D (2005): [Epidemiology of Sudden Cardiac Death]. Herzschrittmacherther Elektrophysiol 16: 73-77.

Baan J, van d, V, de Bruin HG, Smeenk GJ, Koops J, van Dijk AD, Temmerman D, Senden J, Buis B (1984): Continuous Measurement of Left Ventricular Volume in Animals and Humans by Conductance Catheter. Circulation 70: 812-823.

Bardy GH, Lee KL, Mark DB, Poole JE, Packer DL, Boineau R, Domanski M, Troutman C, Anderson J, Johnson G, McNulty SE, Clapp-Channing N, vidson-Ray LD, Fraulo ES, Fishbein DP, Luceri RM, Ip JH (2005): Amiodarone or an Implantable Cardioverter-Defibrillator for Congestive Heart Failure. N Engl J Med 352: 225-237.

Berg RA, Kern KB, Zuercher M (2008): Post-Cardiac Arrest Myocardial Dysfunction: Adding Insult to Injury. Crit Care Med $\underline{36}$ : 351-352.

Bernard SA, Gray TW, Buist MD, Jones BM, Silvester W, Gutteridge G, Smith K (2002): Treatment of Comatose Survivors of Out-of-Hospital Cardiac Arrest With Induced Hypothermia. N Engl J Med 346: 557-563.

Boddicker KA, Zhang Y, Zimmerman MB, Davies LR, Kerber RE (2005): Hypothermia Improves Defibrillation Success and Resuscitation Outcomes From Ventricular Fibrillation. Circulation 111: 3195-3201. 
Boyer NH, Gerstein MM (1977): Induced Hypothermia in Dogs With Acute Myocardial Infarction and Shock. J Thorac Cardiovasc Surg 74: 286-294.

Buckberg GD, Brazier JR, Nelson RL, Goldstein SM, McConnell DH, Cooper N (1977): Studies of the Effects of Hypothermia on Regional Myocardial Blood Flow and Metabolism During Cardiopulmonary Bypass. I. The Adequately Perfused Beating, Fibrillating, and Arrested Heart. J Thorac Cardiovasc Surg $\underline{73}$ : 87-94.

Burkhoff D, Mirsky I, Suga H (2005): Assessment of Systolic and Diastolic Ventricular Properties Via Pressure-Volume Analysis: a Guide for Clinical, Translational, and Basic Researchers. Am J Physiol Heart Circ Physiol 289: H501-H512.

Dae MW, Gao DW, Ursell PC, Stillson CA, Sessler DI (2003): Safety and efficacy of endovascular cooling and rewarming for induction and reversal of hypothermia in human-sized pigs. Stroke 34: 734-738.

Dalrymple-Hay MJ, Deakin CD, Knight H, Edwards JC, Keeton B, Salmon AP, Monro JL (1999): Induced Hypothermia As Salvage Treatment for Refractory Cardiac Failure Following Paediatric Cardiac Surgery. Eur J Cardiothorac Surg 15: 515-518.

Edman KA, Mattiazzi A, Nilsson E (1974): The Influence of Temperature on the Force-Velocity Relationship in Rabbit Papillary Muscle. Acta Physiol Scand 90: 750-756.

Fischer UM, Cox CS, Jr., Laine GA, Mehlhorn U, Allen SJ (2005): Mild Hypothermia Impairs Left Ventricular Diastolic but Not Systolic Function. J Invest Surg 18: 291-296. 
Fukunami M, Hearse DJ (1989): The Inotropic Consequences of Cooling: Studies in the Isolated Rat Heart. Heart Vessels $\underline{5}$ : 1-9.

Goldberg LI (1958): Effects of hypothermia on contractility of the intact dog heart. Am J Physiol 194 (1): 92-98

Gonzalez MM, Berg RA, Nadkarni VM, Vianna CB, Kern KB, Timerman S, Ramires JA (2008): Left Ventricular Systolic Function and Outcome After inHospital Cardiac Arrest. Circulation 117: 1864-1872.

Greene PS, Cameron DE, Mohlala ML, Dinatale JM, Gardner TJ (1989): Systolic and Diastolic Left Ventricular Dysfunction Due to Mild Hypothermia. Circulation 80: III44-III48.

Hannon JP, Bossone CA, Wade CE (1990): Normal Physiological Values for Conscious Pigs Used in Biomedical Research. Lab Anim Sci 40:293-298.

Hay I, Rich J, Ferber P, Burkhoff D, Maurer MS (2005): Role of Impaired Myocardial Relaxation in the Production of Elevated Left Ventricular Filling Pressure. Am J Physiol Heart Circ Physiol 288: H1203-H1208.

Henderson AH, Cattell MR (1976): Length-Induced Changes in Activation During Contraction. A Study of Mechanical Oscillations in StrontiumMediated Contractions of Cat and Frog Heart Muscle. Circ Res 38: 289-296.

Heusch G, Schulz R, Rahimtoola SH (2005): Myocardial Hibernation: a Delicate Balance. Am J Physiol Heart Circ Physiol 288: H984-H999. 
Hiranandani N, Varian KD, Monasky MM, Janssen PM (2006): FrequencyDependent Contractile Response of Isolated Cardiac Trabeculae Under Hypo-, Normo-, and Hyperthermic Conditions. J Appl Physiol 100: 17271732.

Inesi G, Watanabe S (1967): Temperature Dependence of ATP Hydrolysis and Calcium Uptake by Fragmented Sarcoplasmic Membranes. Arch Biochem Biophys 121: 665-671.

Jung W, Andresen D, Block M, Bocker D, Hohnloser SH, Kuck KH, Sperzel J (2006): [Guidelines for the Implantation of Defibrillators.]. Clin Res Cardiol 95: 696-708.

Kass DA, Bronzwaer JG, Paulus WJ (2004): What Mechanisms Underlie Diastolic Dysfunction in Heart Failure? Circ Res 94: 1533-1542.

Kern KB, Hilwig RW, Rhee KH, Berg RA (1996): Myocardial Dysfunction After Resuscitation From Cardiac Arrest: an Example of Global Myocardial Stunning. J Am Coll Cardiol 28: 232-240.

Klein HU (2006): [Prevention of Sudden Cardiac Death]. Internist (Berl) 47: 1040, 1042-1049.

Kuhn LA, Apter R, Weinrauch H, Jacobson TE, Meltzer L (1963): Hemodynamic and metabolic effects of skin and blood-stream cooling in experimental myocardial infarction with shock. Am J Cardiol 12: 795-801.

Kusuoka H, Ikoma Y, Futaki S, Suga H, Kitabatake A, Kamada T, Inoue M (1991): Positive Inotropism in Hypothermia Partially Depends on an Increase in Maximal $\mathrm{Ca}(2+)$-Activated Force. Am J Physiol 261: H1005-H1010. 
Langendorff O (1897): Über Den Einfluss Von Wärme Und Kälte Auf Das Herz Der Warmblütigen Tiere. Pflügers Arch 61: 355-400.

Lee JA, Allen DG (1997): Calcium Sensitisers: Mechanisms of Action and Potential Usefulness As Inotropes. Cardiovasc Res 36: 10-20.

Leite-Moreira AF, Gillebert TC (2000): The Physiology of Left Ventricular Pressure Fall. Rev Port Cardiol 19: 1015-1021.

Lewis ME, Al Khalidi AH, Townend JN, Coote J, Bonser RS (2002): The Effects of Hypothermia on Human Left Ventricular Contractile Function During Cardiac Surgery. J Am Coll Cardiol 39: 102-108.

Liu B, Wohlfart B, Johansson BW (1990): Effects of Low Temperature on Contraction in Papillary Muscles From Rabbit, Rat, and Hedgehog. Cryobiology 27: 539-546.

Mattheussen M, Mubagwa K, Van Aken H, Wusten R, Boutros A, Flameng W (1996): Interaction of Heart Rate and Hypothermia on Global Myocardial Contraction of the Isolated Rabbit Heart. Anesth Analg 82: 975-981.

Monroe RG, Strang RH, Lafarge CG, Levy J (1964): Ventricular performance, pressure-volume relationships, and $\mathrm{O} 2$ consumption during hypothermia. Am J Physiol 206: 67-73.

Nakae Y, Fujita S, Namiki A (2001): Isoproterenol Enhances Myofilament $\mathrm{Ca}(2+)$ Sensitivity During Hypothermia in Isolated Guinea Pig Beating Hearts. Anesth Analg 93: 846-852. 
National Research Council: Guide for the Care and Use of Laboratory Animals, Veröffentlichung des National Institute of Health Nr. 85-23, überarbeitet 1996; National Academy Press Washington D.C., 1996

Nishimura Y, Naito Y, Nishioka T, Okamura Y (2005): The Effects of Cardiac Cooling Under Surface-Induced Hypothermia on the Cardiac Function in the in Situ Heart. Interact Cardiovasc Thorac Surg 4: 101-105.

Nolan JP, Morley PT, Vanden Hoek TL, Hickey RW, Kloeck WG, Billi J, Bottiger BW, Morley PT, Nolan JP, Okada K, Reyes C, Shuster M, Steen PA, Weil MH, Wenzel V, Hickey RW, Carli P, Vanden Hoek TL and Atkins D (2003): Therapeutic Hypothermia After Cardiac Arrest: an Advisory Statement by the Advanced Life Support Task Force of the International Liaison Committee on Resuscitation. Circulation 108: 118-121.

Nolan JP, Deakin CD, Soar J, Bottinger BW, Smith G (2005): European Resuscitation Council guidelines for resuscitation 2005. Section 4. Adult advanced life support. Resuscitation 67: 39-86

Oung CM, English M, Chiu RC, Hinchey EJ (1992): Effects of hypothermia on hemodynamic responses to dopamine and dobutamine. J Trauma 33: 671678

Perez-de-Sa V, Roscher R, Cunha-Goncalves D, Larsson A, Werner O (2002): Mild Hypothermia Has Minimal Effects on the Tolerance to Severe Progressive Normovolemic Anemia in Swine. Anesthesiology 97: 1189-1197. 
Remensnyder JP, Austen WG (1965): Diastolic pressure-volume relastionships of the left ventricle during hypothermia. J Thorac Cardiovasc Surg 49: 339-351.

Rittenhouse EA, Ito CS, Mohri H, Merendino KA (1971): Circulatory dynamics during surface-induced deep hypothermia and after cardiac arrest for one hour. J Thorac Cardiovasc Surg 61: 359-369

Schmidt-Schweda S (2004): Cool-Shock I Study: Mild Hypothermia As Positive Inotropic Intervention in Cardiogenic Shock. Circulation 110: 1693.

Shattock MJ, Bers DM (1987): Inotropic Response to Hypothermia and the Temperature-Dependence of Ryanodine Action in Isolated Rabbit and Rat Ventricular Muscle: Implications for Excitation-Contraction Coupling. Circ Res 61: 761-771.

Steendijk P, Baan J (2000): Comparison of Intravenous and Pulmonary Artery Injections of Hypertonic Saline for the Assessment of Conductance Catheter Parallel Conductance. Cardiovasc Res 46: 82-89.

Steendijk P, Tulner SA, Wiemer M, Beasdale RA, Bax JJ, van der Wall EE, Vogt J, Schalij MJ (2004): Pressure-volume measurements during cardiac resynchronization therapy. Eur heart J Suppl $\underline{6}$ : D35-D42.

Stowe DF, Camara AK, Heisner JS, Aldakkak M, Harder DR (2007): TenHour Preservation of Guinea Pig Isolated Hearts Perfused at Low Flow With Air-Saturated Lifor Solution at $26^{\circ} \mathrm{C}$ : Comparison to ViaSpan Solution. Am J Physiol Heart Circ Physiol 293: H895-H901. 
Suga H, Hisano R, Goto Y, Yamada O, Igarashi Y (1983): Effect of Positive Inotropic Agents on the Relation Between Oxygen Consumption and Systolic Pressure Volume Area in Canine Left Ventricle. Circ Res 53: 306-318.

Suga $\mathrm{H}$, Goto $\mathrm{Y}$, Igarashi $\mathrm{Y}$, Yasumura $\mathrm{Y}$, Nozawa T, Futaki S, Tanaka N (1988): Cardiac Cooling Increases Emax Without Affecting Relation Between O2 Consumption and Systolic Pressure-Volume Area in Dog Left Ventricle. Circ Res 63: 61-71.

Templeton GH, Wildenthal K, Willerson JT, Reardon WC (1974): Influence of Temperature on the Mechanical Properties of Cardiac Muscle. Circ Res 34: 624-634.

The hypothermia after cardiac arrest study group (2002): Mild Therapeutic Hypothermia to Improve the Neurologic Outcome After Cardiac Arrest. N Engl J Med 346: 549-556.

Tveita T, Mortensen E, Hevroy O, Refsum H, Ytrehus K (1994): Experimental hypothermia: effects of core cooling and rewarming on hemodynamics, coronary blood flow, and myocardial metabolism in dogs. Anesth Analg $\underline{79}$ : 212-218

Tveita T, Ytrehus K, Myhre ES, Hevroy O (1998): Left Ventricular Dysfunction Following Rewarming From Experimental Hypothermia. J Appl Physiol 85: 2135-2139.

Weisser J, Martin J, Bisping E, Maier LS, Beyersdorf F, Hasenfuss G, Pieske B (2001): Influence of Mild Hypothermia on Myocardial Contractility and Circulatory Function. Basic Res Cardiol 96: 198-205. 
Wolfrum S, Radke PW, Pischon T, Willich SN, Schunkert H, Kurowski V (2007): Mild Therapeutic Hypothermia After Cardiac Arrest - a Nationwide Survey on the Implementation of the ILCOR Guidelines in German Intensive Care Units. Resuscitation 72: 207-213.

Yahagi N, Kumon K, Watanabe $\mathrm{Y}$, Tanigami $\mathrm{H}$, Haruna M, Hayashi $\mathrm{H}$, Imanaka H, Takeuchi M, Ohashi Y, Takamoto S (1998): Value of Mild Hypothermia in Patients Who Have Severe Circulatory Insufficiency Even After Intra-Aortic Balloon Pump. J Clin Anesth 10:120-125.

Yeatman LA, Jr., Parmley WW, Sonnenblick EH (1969): Effects of Temperature on Series Elasticity and Contractile Element Motion in Heart Muscle. Am J Physiol 217:1030-1034.

Zhao H, Steinberg GK, Sapolsky RM (2007): General Versus Specific Actions of Mild-Moderate Hypothermia in Attenuating Cerebral Ischemic Damage. J Cereb Blood Flow Metab 27:1879-1894. 


\section{Publikation zum Inhalt der Dissertation}

Der Inhalt dieser Arbeit wurde in dem Artikel "Cardiac function during mild hypothermia in pigs: increased inotropy at the expense of diastolic dysfunction" (Post H, Schmitto JD, Steendijk P, Christoph J, Holland R, Wachter R, Schöndube FW, Pieske B) erschienen in Acta Physiologica 2010, 199, 43-52, veröffentlicht. 